\title{
A general tensor representation framework for cross-view gait recognition
}

\author{
Xianye Ben ${ }^{\mathrm{a}, *}$, Peng Zhang, ${ }^{\mathrm{a}, \mathrm{b}}$, Zhihui Lai ${ }^{\mathrm{c}}$, Rui Yan $^{\mathrm{d}}$, Xinliang Zhai ${ }^{\mathrm{a}}$, Weixiao \\ Menge \\ ${ }^{a}$ School of Information Science and Engineering, Shandong University, Qingdao 266237, \\ China \\ ${ }^{b}$ School of Electrical and Data Engineering, University of Technology Sydney, Sydney, NSW \\ 2007, Australia \\ ${ }^{c}$ College of Computer Science and Software Engineering, Shenzhen University, Shenzhen \\ 518060, China \\ ${ }^{d}$ Computer Science Department, Rensselaer Polytechnic Institute, Troy, NY 12180, USA \\ ${ }^{e}$ School of Electronics and Information Engineering, Harbin Institute of Technology, Harbin \\ 150080, China
}

\begin{abstract}
Tensor analysis methods have played an important role in identifying human gaits using high dimensional data. However, when view angles change, it becomes more and more difficult to recognize cross-view gait by learning only a set of multi-linear projection matrices. To address this problem, a general tensor representation framework for cross-view gait recognition is proposed in this paper. There are three criteria of tensorial coupled mappings in the proposed framework. (1) Coupled multi-linear locality-preserved criterion (CMLP) aims to detect the essential tensorial manifold structure via preserving local information. (2) Coupled multi-linear marginal fisher criterion (CMMF) aims to encode the intra-class compactness and inter-class separability with local relationships. (3) Coupled multi-linear discriminant analysis criterion (CMDA) aims to minimize the intra-class scatter and maximize the inter-class scatter. For the three tensor algorithms for cross-view gaits, two sets of multi-linear projection matrices are iteratively learned using alternating projection optimization procedures. The proposed methods are compared with the recently published cross-view gait
\end{abstract}

\footnotetext{
*Corresponding author. Tel.:+86 15254130623; Fax: +86 15254130623

Email address: benxianye@gmail.com (Xianye Ben)
} 
recognition approaches on CASIA(B) and OU-ISIR gait database. The results demonstrate that the performances of the proposed methods are superior to existing state-of-the-art cross-view gait recognition approaches.

Keywords: Gait recognition, cross-view gait, tensor representation, framework

\section{Introduction}

The demand to recognize and authenticate individuals using biometrics has been rising due to its broad applications in security and surveillance. During past decades, many biometrics have been applied to practice such as face, fin-

5 gerprint and iris. These biometrics are unique from person to person, which is essential to quickly identify the target's identity. Different from these biometrics, i.e., face, fingerprint, vein, iris, ear, hand shape, palm print, retina and lip, gait is a kind of soft biometric which aims to recognize one's identity by his unique walking patterns. It is more potential than the biological characteristics in the surveillance field due to the advantage of gait recognition [1, 2] lies in the fact that it can be efficiently recognized at a distance without subjects' cooperation.

The popular gait recognition methods can be roughly classified into the following two categories: model-based [3] and motion-based approaches [4, 5, 6]. 15 Model-based approaches can extract the gait features robustly and avoid the noise interference problem. The changes all over the body can be characterized by a short vector. It is possible for gait recognition to obtain a good performance if the model is established accurately. However, the modelling and its matching processes are both complex. Compared to model-based methods, motion-based approaches avoid the complex modelling which can characterize the motion patterns of human body without fitted model parameters. Due to the benefits, motion-based approaches attract more attention recent years. However, a challenge to motion-based gate recognition is the cross-view issue [7, 8]. This is because motion pattern changes dramatically as the viewing di${ }_{25}$ rections move, even though when it is the same subject's gait. This is the main 
reason why most state-of-the-art motion-based gate recognition method do not perform well [9, 10, 11, 12]. Basically, there is a trend that the larger the variation of viewing direction is, the worse the recognition performance is. Thus, the core research question of this paper is how can we find robust and discriminative representations, such that they can enlarge the discrimination between different subjects, and meanwhile compact the variations of the same subject?

Previous methods try to bridge the view biases by constructing $3 \mathrm{D}$ model and performing view transformation model, however, the former is computationally complex and the latter does not consider discriminability. Data-driven CNN 35 approaches achieve significant success in many fields [13, 14, 15, 16], which can be also applied to gait recognition. Due to its powerful representation ability, CNN can extract view invariant features. However, limited labelled data available easily causes the CNN model over-fitting. In another aspect, CNN model highly relies on expensive GPU hardware to accelerate the training 40 speed. Different from them, tensors are higher order generalizations of matrices [17], which is helpful to reduce the small sample size problem in discriminative subspace selection. They have been successfully applied to gait recognition under a fixed angle of view.

Inspired by success of tensor representation, this paper presents a novel gen45 eral tensor representation framework for cross-view gait recognition. Our formulations model the gait data as a tensor and seek three robust and tensorial discriminative representations by tensor analysis. Our framework can leverage structure information and reduce the number of parameters used to model the cross-view gait recognition. We present three novel criteria of tensorial coupled mappings. First, by preserving local information, we obtain a common subspace that best detects the essential gait manifold structure. Second, by encoding the intra-class compactness and inter-class separability with local relationships, we present coupled multi-linear marginal fisher criterion. Third, by minimizing the intra-class scatter of cross-view gaits, and simultaneously maximizing the inter-

${ }_{55}$ class scatter, we propose coupled multi-linear discriminant analysis criterion. These three tensor alignment algorithms of cross-view gaits are achieved by 
alternating projection optimization procedures. The flourishing of cross-view gait recognition methods depends largely on well-established multi-view gait databases, such as CASIA(B) [18] and OU-ISIR [19]. To the best of our knowledge, our work is the first attempt to address the cross-view gait recognition within a framework of tensor representations. The key contributions of our work can be summarized as follows:

(1) We propose to model cross-view gait data as tensors and develop a novel framework of cross-view gait recognition by tensor representations.

(2) We present three novel criteria of tensorial coupled mappings with their tensor alignment algorithms of cross-view gaits.

(3) We systematically evaluate our methods on both the largest number of cross-views gait database and the largest population gait database.

The remainder of this paper is organized as follows: Section 2 briefly reviews some related works. Section 3 presents our general tensor representation framework for cross-view gait recognition. After that, Section 4 proposes 3 criteria of tensorial coupled mappings with their tensor alignment algorithms of cross-view gaits. Then, Section 5 demonstrates the experimental results on both CASIA(B) and OU-ISIR gait database. Finally, this paper is concluded 75 in Section 6 .

\section{Related work}

In this section, we give a brief literature review of related topics to our work, i.e., cross-view gait recognition and tensor representation for gait analysis.

\subsection{Cross-view gait recognition}

Several related work tries to tackle this cross-view gait recognition problem, which can be categories into three classes. The first class of work focuses on constructing 3D gait information via panoramic or multiple calibrated cameras $[10,20,21,22]$. These 3D-based methods are usually set-up with complicated 
environment of controlled multi-cameras, which may not be available in practice. Even if it is available, its practical application can be adversely impacted by the computation complexity. The second category is based on view transformation model (VTM). This includes single value decomposition (SVD) and regression, which have been massively deployed to generate gait features with the information from the other view [23, 24, 25, 26, 27, 28, 29, 30, 31, 32, 33.

90 Although these methods minimize the errors between the transformed features and original gaits features, they do not consider the discrimination capability. The third category is to extract view-invariant gait feature. Generally, it infers a view-invariant gait feature among cross-view gait data. For example, in [34], Goffredo et al. proposed a self-calibration of limbs' pose in the image reference 95 system. However, this method can only coarsely estimate the limbs' pose when the view of input gait is very different from the registered or front view gaits. To alleviate this problem, domain transformation [35, 36, 37, 38], metric learning [39], and deep CNNs [40, 41, 42] have been introduced recently. Especially, deep CNNs have achieved encouraging recognition accuracy on the cross-view task due to its powerful representation ability. The premise of using deep CNNs requires to a large quantity of labeled training data efficiently, however, the limited gait data available restricts its application.

\subsection{Tensor representation for gait analysis}

A variety of multi-linear subspace learning approaches based on tensor representation have been applied to gait analysis, which can not only extract spatial-temporal gait information but also avoid small size sample problem. For example, Lu et al. [43] proposed multi-linear principal component analysis (MPCA) to capture most of the original tensorial input variation. Then, they extend MPCA to Uncorrelated multi-linear principal component analysis ${ }_{110}$ (UMPCA) [44], which can produce uncorrelated features while capturing most of the variation in the original tensorial input. However, the above algorithms only concentrate on unsupervised dimension reduction instead of discriminative feature extraction and classification. Therefore, multi-linear discriminant 
analysis (MLDA) approaches [45, 46, 47] are proposed for gait feature extrac-

framework for cross-view gait recognition is still lack of study.

\section{A general tensor representation framework for cross-view gait recog- nition}

\subsection{Cross-view gait data multi-linear transformation}

Human gait samples are usually represented by second-order tensor or higherorder tensor. Given two sets of training gait tensorial samples $\left\{\mathcal{X}_{i} \in \mathbb{R}^{H_{1} \times H_{2} \times \cdots \times H_{N}}\right.$, $\left.i=1,2, \ldots, M_{\theta}\right\}$ and $\left\{\mathcal{Y}_{j} \in \mathbb{R}^{L_{1} \times L_{2} \times \cdots \times L_{N}}, j=1,2, \ldots, M_{\vartheta}\right\}$ from two views $\theta$ and $\vartheta$, where $H_{n}$ and $L_{n}$ are mode- $n$ dimensions for views $\theta$ and $\vartheta$, respectively. $M_{\theta}$ and $M_{\vartheta}$ are the numbers of gait tensorial samples for views $\theta$ and $\vartheta$, respectively. Generally, both share a consistent one-to-one match between two views, namely, $M_{\theta}=M_{\vartheta}=M$. Now our goal is to find transformation functions $f_{\theta}\left(\mathcal{X}_{i}\right)$ and $f_{\vartheta}\left(\mathcal{Y}_{j}\right)$ to make gait data under different views project 
Table 1: Similarity relationship between $\mathcal{X}_{i}$ and $\mathcal{Y}_{i}$

\begin{tabular}{|c|c|c|c|}
\hline $\begin{array}{l}\text { Side } \\
\text { information }\end{array}$ & $w_{i j}=\{$ & $\begin{array}{l}1,\left\{\mathcal{X}_{i}, \mathcal{X}_{j}\right\} \in \mathbb{C} \\
0, \text { else }\end{array}$ & $\begin{array}{l}\mathcal{X}_{i} \text { and } \mathcal{Y}_{i} \text { belong } \\
\text { to the identical } \\
\text { individual } \mathbb{C} \text {. }\end{array}$ \\
\hline $\begin{array}{l}\text { Cosine } \\
\text { similarity }\end{array}$ & $w_{i j}=$ & $\begin{array}{l}\frac{\operatorname{vec}\left(\mathcal{X}_{i}\right) \cdot \operatorname{vec}\left(\mathcal{X}_{j}\right)}{\left\|\operatorname{vec}\left(\mathcal{X}_{i}\right)\right\|\left\|\operatorname{vec}\left(\mathcal{X}_{j}\right)\right\|}, \text { if } \mathcal{X}_{i} \in N_{k}\left(\mathcal{X}_{j}\right) \text { or } \mathcal{X}_{j} \in N_{k}\left(\mathcal{X}_{i}\right) \\
0, \text { else }\end{array}$ & $\begin{array}{l}v e c(\cdot) \text { denotes a } \\
\text { vectorization operator. } \\
N_{k}(\cdot) \text { denotes } \\
k \text { nearest neighbor. }\end{array}$ \\
\hline $\begin{array}{l}\text { Gaussian } \\
\text { similarity }\end{array}$ & $w_{i j}=$ & $\begin{array}{l}\exp \left(-\left\|\operatorname{vec}\left(\mathcal{X}_{i}\right)-\operatorname{vec}\left(\mathcal{X}_{j}\right)\right\|^{2} / t\right), \text { if } \mathcal{X}_{i} \in N_{k}\left(\mathcal{X}_{j}\right) \text { or } \mathcal{X}_{j} \in N_{k}\left(\mathcal{X}_{i}\right) \\
0, \text { else }\end{array}$ & $\begin{array}{l}t \text { denotes Gaussian } \\
\text { variance parameter. }\end{array}$ \\
\hline
\end{tabular}

into a common space and measure their similarity. The objective of a general tensor representation framework for cross-view gait recognition is to find a pair of multi-linear transformation matrices $\left\{\mathbf{U}_{n} \in H_{n} \times F_{n}, n=1, \ldots, N\right\}$ and $\left\{\mathbf{V}_{n} \in L_{n} \times F_{n}, n=1, \ldots, N\right\}$ to project cross-view gait samples into a common lower-dimensional tensorial subspace $F_{1} \otimes F_{2} \otimes \cdots \otimes F_{N}$ from both of original tensorial space $H_{1} \otimes H_{2} \otimes \cdots \otimes H_{N}$ and $L_{1} \otimes L_{2} \otimes \cdots \otimes L_{N}$ respectively. Thus,

$$
\begin{aligned}
& \mathcal{A}_{i}=\mathcal{X}_{i} \times{ }_{1} \mathbf{U}_{1}^{\top} \times{ }_{2} \mathbf{U}_{2}^{\top} \cdots \times{ }_{N} \mathbf{U}_{N}^{\top}=\mathcal{X}_{i} \prod_{k=1}^{N} \times{ }_{k} \mathbf{U}_{k}^{\top}, \\
& \mathcal{B}_{j}=\mathcal{Y}_{j} \times{ }_{1} \mathbf{V}_{1}^{\top} \times{ }_{2} \mathbf{V}_{2}^{\top} \cdots \times{ }_{N} \mathbf{V}_{N}^{\top}=\mathcal{Y}_{j} \prod_{k=1}^{N} \times{ }_{k} \mathbf{V}_{k}^{\top},
\end{aligned}
$$

150

where $\mathcal{A}_{i}$ and $\mathcal{B}_{j}$ are projected tensor features for the views $\theta$ and $\vartheta$, and $\mathcal{A}_{i}, \mathcal{B}_{j} \in$ $\mathbb{R}^{F_{1} \times F_{2} \times \cdots \times F_{N}}, i, j=1, \ldots, M . F_{n}$ is the mode- $n$ dimension of the projected tensor features, and $F_{n} \leq \min \left(H_{n}, L_{n}\right)$.

\subsection{The similarity of cross-view gait data}

To simplify the calculation on the similarity between $\mathcal{X}_{i}$ and $\mathcal{Y}_{j}$ across view, we define

$$
\operatorname{sim}\left(\mathcal{X}_{i}, \mathcal{Y}_{j}\right)=\left\|\mathcal{A}_{i}-\mathcal{B}_{j}\right\|_{F}^{2} w_{i j}
$$

$w_{i j}$ is the similarity relationship between $\mathcal{X}_{i}$ and $\mathcal{X}_{j}$, which can be calculated according to side information constraint, Gaussian similarity or Cosine similarity [52]. The similarity presented in Table 1 can be used in this paper. 
Table 2: The criteria, the alignment representation and the objective function used in the proposed framework

\begin{tabular}{|c|c|c|}
\hline Criteria & Alignment representation & Objective function for mode $n$ \\
\hline $\begin{array}{l}\text { Coupled } \\
\text { multi-linear } \\
\text { locality-preserved } \\
\text { criterion (CMLP) }\end{array}$ & {$\left[\begin{array}{cc}\mathbf{D}_{1} \otimes \mathbf{I} & -\mathbf{W} \otimes \mathbf{I} \\
-\mathbf{W}^{\top} \otimes \mathbf{I} & \mathbf{D}_{2} \otimes \mathbf{I}\end{array}\right.$} & $\arg \min _{\mathbf{U}_{n}, \mathbf{V}_{n}} \sum_{i j}\left\|\mathbf{U}_{n}^{\top} \mathbf{X}_{i(n)} \tilde{\mathbf{U}}_{n}-\mathbf{V}_{n}^{\top} \mathbf{Y}_{j(n)} \tilde{\mathbf{V}}_{n}\right\|_{F}^{2} w_{i j}$ \\
\hline $\begin{array}{l}\text { Coupled } \\
\text { multi-linear } \\
\text { marginal fisher } \\
\text { criterion (CMMF) }\end{array}$ & {$\left[\begin{array}{cc}\overline{\mathbf{D}}_{1} \otimes \mathbf{I} & -\overline{\mathbf{W}} \otimes \mathbf{I} \\
-\overline{\mathbf{W}}^{\top} \otimes \mathbf{I} & \overline{\mathbf{D}}_{2} \otimes \mathbf{I} \\
\tilde{\mathbf{D}}_{1} \otimes \mathbf{I} & -\tilde{\mathbf{W}} \otimes \mathbf{I} \\
-\tilde{\mathbf{W}}^{\top} \otimes \mathbf{I} & \tilde{\mathbf{D}}_{2} \otimes \mathbf{I}\end{array}\right.$} & $\arg \min _{\mathbf{U}_{n}, \mathbf{V} n} \frac{\sum_{\pi_{i}=\pi_{j}}\left\|\mathbf{U}_{n}^{\top} \mathbf{x}_{i(n)} \tilde{\mathbf{U}}_{n}-\mathbf{V}_{n}^{\top} \mathbf{Y}_{j(n)} \tilde{\mathbf{V}}_{n}\right\|_{F}^{2} \bar{w}_{i j}}{\sum_{\pi_{i} \neq \pi_{j}}\left\|\mathbf{U}_{n}^{\top} \mathbf{x}_{i(n)} \tilde{\mathbf{U}}_{n}-\mathbf{V}_{n}^{\top} \mathbf{Y}_{j(n)} \tilde{\mathbf{V}}_{n}\right\|_{F}^{2} \tilde{w}_{i j}}$ \\
\hline $\begin{array}{l}\text { Coupled } \\
\text { multi-linear } \\
\text { discriminant } \\
\text { analysis criterion } \\
\text { (CMDA) }\end{array}$ & {$\left[\begin{array}{cc}\mathbf{I}-\mathbf{K} \otimes \mathbf{I} & \mathbf{0} \\
\mathbf{0} & \mathbf{I}-\mathbf{K} \otimes \mathbf{I} \\
\mathbf{K} \otimes \mathbf{I}-\frac{1}{M}\left(\mathbf{e} \mathbf{e}^{\top}\right) \otimes \mathbf{I} \\
\mathbf{0} & \mathbf{K}\end{array}\right.$} & $\arg \max _{\mathbf{U}_{n}, \mathbf{V}_{n}} \frac{\sum_{c=1}^{C}\left(\begin{array}{c}N_{c}\left\|\mathbf{U}_{n}^{\top} \overline{\mathbf{X}}_{(n)}^{(c)} \tilde{\mathbf{U}}_{n}-\mathbf{U}_{n}^{\top} \overline{\mathbf{X}}_{(n)} \tilde{\mathbf{U}}_{n}\right\|_{F}^{2} \\
+N_{c}\left\|\mathbf{V}_{n}^{\top} \overline{\mathbf{Y}}_{(n)}^{(c)} \tilde{\mathbf{V}}_{n}-\mathbf{V}_{n}^{\top} \overline{\mathbf{Y}}_{(n)} \tilde{\mathbf{V}}_{n}\right\|_{F}^{2}\end{array}\right.}{\sum_{c=1}^{C}\left(\begin{array}{l}\sum_{i=1}^{N_{c}}\left\|\mathbf{U}_{n}^{\top} \mathbf{X}_{i(n)}^{(c)} \tilde{\mathbf{U}}_{n}-\mathbf{U}_{n}^{\top} \overline{\mathbf{X}}_{(n)}^{(c)} \tilde{\mathbf{U}}_{n}\right\|_{F}^{2} \\
+\sum_{j=1}^{N_{c}}\left\|\mathbf{V}_{n}^{\top} \mathbf{Y}_{j(n)}^{(c)} \tilde{\mathbf{V}}_{n}-\mathbf{V}_{n}^{\top} \overline{\mathbf{Y}}_{(n)}^{(c)} \tilde{\mathbf{V}}_{n}\right\|_{F}^{2}\end{array}\right.}$ \\
\hline
\end{tabular}

The mode- $n$ unfoldings of $\mathcal{A}_{i}$ and $\mathcal{B}_{j}$ can be derived as

$$
\mathbf{A}_{i(n)}=\mathbf{U}_{n}^{\top} \mathbf{X}_{i(n)}\left(\mathbf{U}_{N} \otimes \ldots \otimes \mathbf{U}_{n+1} \otimes \mathbf{U}_{n-1} \otimes \ldots \otimes \mathbf{U}_{1}\right)=\mathbf{U}_{n}^{\top} \mathbf{X}_{i(n)} \tilde{\mathbf{U}}_{n}
$$

$$
\mathbf{B}_{j(n)}=\mathbf{V}_{n}^{\top} \mathbf{Y}_{j(n)}\left(\mathbf{V}_{N} \otimes \ldots \otimes \mathbf{V}_{n+1} \otimes \mathbf{V}_{n-1} \otimes \ldots \otimes \mathbf{V}_{1}\right)=\mathbf{V}_{n}^{\top} \mathbf{Y}_{j(n)} \tilde{\mathbf{V}}_{n}
$$

where $\tilde{\mathbf{U}}_{n}=\prod_{k=1, k \neq n}^{N} \otimes \mathbf{U}_{k}, \tilde{\mathbf{V}}_{n}=\prod_{k=1, k \neq n}^{N} \otimes \mathbf{V}_{k}$. (9) can be written as

$$
\begin{aligned}
& \operatorname{sim}\left(\mathcal{X}_{i}, \mathcal{Y}_{j}\right)=\left\|\mathcal{A}_{i}-\mathcal{B}_{j}\right\|_{F}^{2} w_{i j} \\
& =\operatorname{Tr}\left(\left[\begin{array}{c}
\mathbf{U}_{n} \\
\mathbf{V}_{n}
\end{array}\right]^{\top}\left[\begin{array}{cc}
\mathbf{X}_{i(n)} \tilde{\mathbf{U}}_{n} & \mathbf{0} \\
\mathbf{0} & \mathbf{Y}_{j(n)} \tilde{\mathbf{V}}_{n}
\end{array}\right]\left[\begin{array}{cc}
w_{i j} \cdot \mathbf{I} & -w_{i j} \cdot \mathbf{I} \\
-w_{i j} \cdot \mathbf{I} & w_{i j} \cdot \mathbf{I}
\end{array}\right]\left[\begin{array}{cc}
\mathbf{X}_{i(n)} \tilde{\mathbf{U}}_{n} & \mathbf{0} \\
\mathbf{0} & \mathbf{Y}_{j(n)} \tilde{\mathbf{V}}_{n}
\end{array}\right]^{\top}\left[\begin{array}{c}
\mathbf{U}_{n} \\
\mathbf{V}_{n}
\end{array}\right]\right) \\
& =\operatorname{Tr}\left(\mathbf{P}_{n}^{\top} \mathbf{Z}_{i j}^{(n)} \mathbf{G}_{i j}\left(\mathbf{Z}_{i j}^{(n)}\right)^{\top} \mathbf{P}_{n}\right),
\end{aligned}
$$

where $\mathbf{P}_{n}=\left[\begin{array}{c}\mathbf{U}_{n} \\ \mathbf{V}_{n}\end{array}\right], \mathbf{Z}_{i j}^{(n)}=\left[\begin{array}{cc}\mathbf{X}_{i(n)} \tilde{\mathbf{U}}_{n} & \mathbf{0} \\ \mathbf{0} & \mathbf{Y}_{j(n)} \tilde{\mathbf{V}}_{n}\end{array}\right], \mathbf{G}_{i j}=\left[\begin{array}{cc}w_{i j} \cdot \mathbf{I} & -w_{i j} \cdot \mathbf{I} \\ -w_{i j} \cdot \mathbf{I} & w_{i j} \cdot \mathbf{I}\end{array}\right]$.

The detailed mathematical deductions are put into the Appendix A.

In the following section, we will introduce 3 criteria with tensorial coupled mappings to obtain $\mathbf{U}_{n}$ and $\mathbf{V}_{n}$. Table 2 shows the alignment representation of each criteria used in the proposed framework. 


\subsection{Classification}

We denote $\mathbf{Q}_{1}$ and $\mathbf{Q}_{2}$ be projection matrices trained by the improved metric learning approach [52] respectively for the vectorized cross-view gait data

180

\section{Three criteria of tensorial coupled mappings}

In this section, we introduce three different criteria under the unified tonsorial framework in the last section and analyse the relationship between them.

\subsection{Coupled multi-linear locality-preserved criterion (CMLP)}

185

Preserving local information, tensorial coupled mappings with CMLP criterion aim to learn a couple of multi-linear projection matrices for views $\theta$ and $\vartheta$ to obtain a common subspace that best detects the essential gait manifold structure. The objective function is defined as

$$
\left\{\mathbf{U}_{n}^{*}, \mathbf{V}_{n}^{*}, n=1, \ldots, N\right\}=\underset{\mathbf{U}_{n}, \mathbf{V}_{n}, n=1, \ldots, N}{\arg \min } \sum_{i j}\left\|\mathcal{X}_{i} \prod_{k=1}^{N} \times_{k} \mathbf{U}_{k}^{\top}-\mathcal{Y}_{j} \prod_{k=1}^{N} \times_{k} \mathbf{V}_{k}^{\top}\right\|_{F}^{2} w_{i j}
$$


The above objective function does not have a closed-form solution, so an iterative procedure (see Algorithm 1) is proposed to solve it.

Its mode- $n$ objective function can be written in a trace form as

$$
\begin{aligned}
& \arg \min _{\mathbf{U}_{n}, \mathbf{V}_{n}} J\left(\mathbf{U}_{n}, \mathbf{V}_{n}\right)=\sum_{i j}\left\|\mathbf{U}_{n}^{\top} \mathbf{X}_{i(n)} \tilde{\mathbf{U}}_{n}-\mathbf{V}_{n}^{\top} \mathbf{Y}_{j(n)} \tilde{\mathbf{V}}_{n}\right\|_{F}^{2} w_{i j} \\
& =\operatorname{Tr}\left(\left[\begin{array}{c}
\mathbf{U}_{n} \\
\mathbf{V}_{n}
\end{array}\right]^{\top}\left[\begin{array}{cc}
\mathbf{X}_{(n)} \tilde{\mathbf{U}}_{n} & \mathbf{0} \\
\mathbf{0} & \mathbf{Y}_{(n)} \tilde{\mathbf{V}}_{n}
\end{array}\right]\left[\begin{array}{cc}
\mathbf{D}_{1} \otimes \mathbf{I} & -\mathbf{W} \otimes \mathbf{I} \\
-\mathbf{W}^{\top} \otimes \mathbf{I} & \mathbf{D}_{2} \otimes \mathbf{I}
\end{array}\right]\left[\begin{array}{cc}
\mathbf{X}_{(n)} \tilde{\mathbf{U}}_{n} & \mathbf{0} \\
\mathbf{0} & \mathbf{Y}_{(n)} \tilde{\mathbf{V}}_{n}
\end{array}\right]^{\top}\left[\begin{array}{c}
\mathbf{U}_{n} \\
\mathbf{V}_{n}
\end{array}\right]\right),
\end{aligned}
$$

where $\mathbf{X}_{(n)}=\left[\mathbf{X}_{1(n)}, \mathbf{X}_{2(n)}, \ldots, \mathbf{X}_{M(n)}\right], \mathbf{Y}_{(n)}=\left[\mathbf{Y}_{1(n)}, \mathbf{Y}_{2(n)}, \ldots, \mathbf{Y}_{M(n)}\right], \otimes$ denotes Kronecker product, and $\mathbf{I}$ is a unit matrix, similarity matrix $\mathbf{W}$ is made of $w_{i j}$, and diagonal matrices $\mathbf{D}_{1}$ and $\mathbf{D}_{2}$ are

$$
\mathbf{D}_{1}=\left[\begin{array}{ccc}
\sum_{j} w_{1 j} & \mathbf{0} & \mathbf{0} \\
\mathbf{0} & \ddots & \mathbf{0} \\
\mathbf{0} & \mathbf{0} & \sum_{j} w_{M j}
\end{array}\right], \mathbf{D}_{2}=\left[\begin{array}{ccc}
\sum_{i} w_{i 1} & \mathbf{0} & \mathbf{0} \\
\mathbf{0} & \ddots & \mathbf{0} \\
\mathbf{0} & \mathbf{0} & \sum_{i} w_{i M}
\end{array}\right] .
$$

The detailed mathematical deductions of (11) are put into the Appendix B.

To simplify (11), several auxiliary matrices are defined as follows

$$
\mathbf{P}_{n}=\left[\begin{array}{c}
\mathbf{U}_{n} \\
\mathbf{V}_{n}
\end{array}\right], \mathbf{Z}_{(n)}=\left[\begin{array}{cc}
\mathbf{X}_{(n)} \tilde{\mathbf{U}}_{n} & \mathbf{0} \\
\mathbf{0} & \mathbf{Y}_{(n)} \tilde{\mathbf{V}}_{n}
\end{array}\right], \mathbf{G}=\left[\begin{array}{cc}
\mathbf{D}_{1} \otimes \mathbf{I} & -\mathbf{W} \otimes \mathbf{I} \\
-\mathbf{W}^{\top} \otimes \mathbf{I} & \mathbf{D}_{2} \otimes \mathbf{I}
\end{array}\right] .
$$

Hence, we have the following objective function for mode- $n$

$$
\left\{\mathbf{P}_{n}^{*}, n=1, \ldots, N\right\}=\arg \min _{\mathbf{P}_{n}} \operatorname{Tr}\left(\mathbf{P}_{n}^{\top} \mathbf{Z}_{(n)} \mathbf{G} \mathbf{Z}_{(n)}^{\top} \mathbf{P}_{n}\right)
$$

where $\mathbf{P}_{n}^{*}$ denotes the optimal solution for mode- $n$. Therefore, (11) has been decomposed into $N$ different sub-optimization problems although $2 N$ optimized variables are coupled in a single objective function.

With the orthogonal constraints introduced into the (14) to make $\left\{\mathbf{P}_{n}^{*}, n=1, \ldots, N\right\}$ unique, we derive

$$
\begin{array}{ll}
\left\{\mathbf{P}_{n}^{*}, n=1, \ldots, N\right\}=\arg \min _{\mathbf{P}_{n}} \operatorname{Tr}\left(\mathbf{P}_{n}^{\top} \mathbf{Z}_{(n)} \mathbf{G} \mathbf{Z}_{(n)}^{\top} \mathbf{P}_{n}\right) \\
\text { s.t. } \quad \mathbf{P}_{n}^{\top} \mathbf{P}_{n}=\mathbf{I},
\end{array}
$$




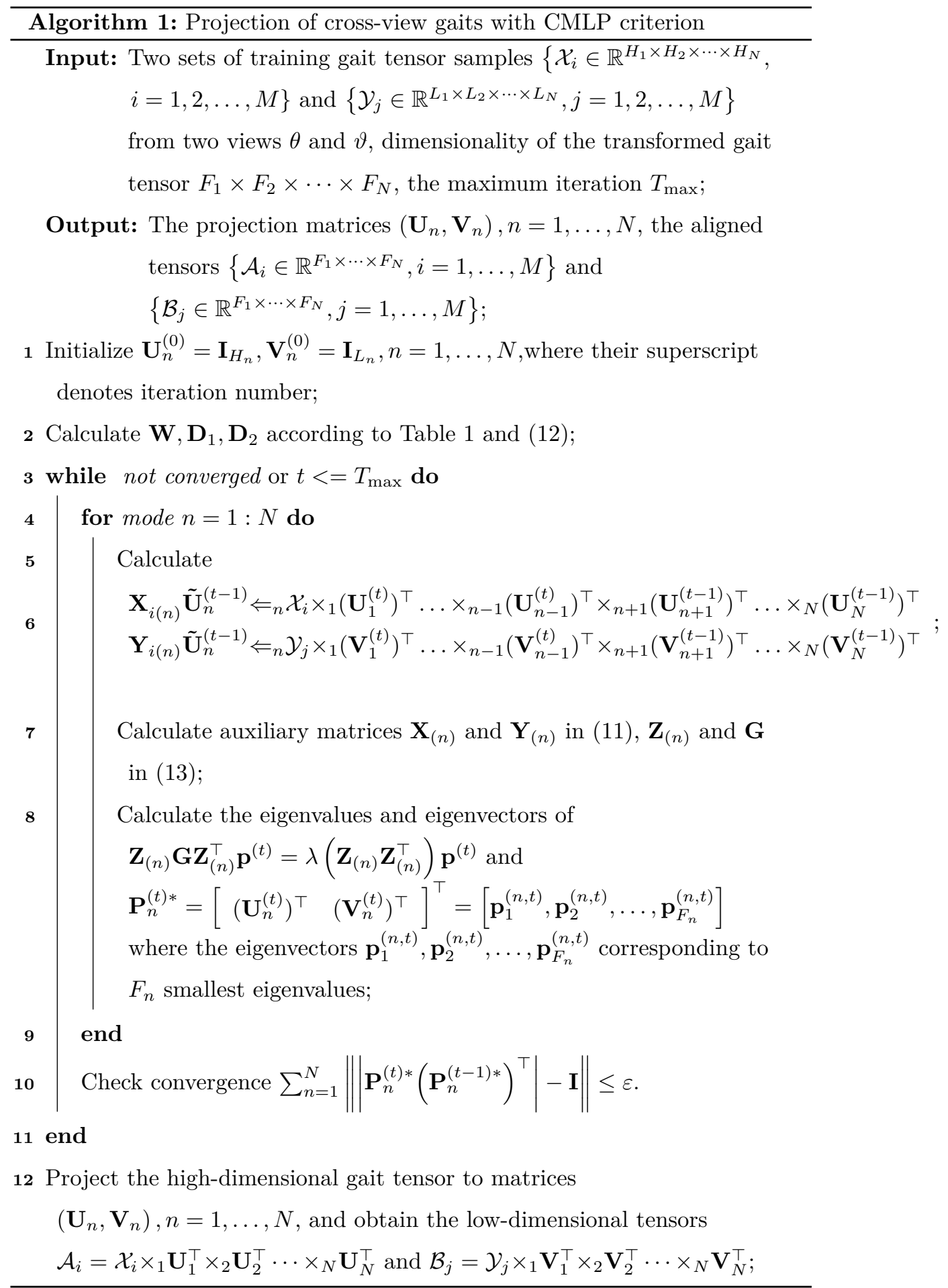


where $\mathbf{I} \in \mathbb{R}^{F_{n} \times F_{n}}$ is a unit matrix, $e \in \mathbb{R}^{2 M K_{n} \times 1}$ is a column vector of ones, $K_{n}=F_{n+1} \times \cdots \times F_{N} \times F_{n-1} \times \cdots \times F_{1}$, the size of a column vector of zeros $\mathbf{0}$ is $F_{n} \times 1$. The solution of 15 are listed in Appendix C

\subsection{Coupled multi-linear marginal fisher criterion (CMMF)}

We proposed CMMF criterion to encode the intra-class compactness and inter-class separability with local relationships. As a result, for each mode $n$, the objective function is

$$
\left\{\mathbf{U}_{n}^{*}, \mathbf{V}_{n}^{*}, n=1, \ldots, N\right\}=\underset{\mathbf{U}_{n}, \mathbf{V}_{n}, n=1, \ldots, N}{\arg \min } \frac{\sum_{\pi_{i}=\pi_{j}}\left\|\mathcal{X}_{i} \times{ }_{1} \mathbf{U}_{1}^{\top} \cdots \times_{N} \mathbf{U}_{N}^{\top}-\mathcal{Y}_{j} \times{ }_{1} \mathbf{V}_{1}^{\top} \cdots \times_{N} \mathbf{V}_{N}^{\top}\right\|_{F}^{2} \bar{w}_{i j}}{\sum_{\pi_{i} \neq \pi_{j}}\left\|\mathcal{X}_{i} \times_{1} \mathbf{U}_{1}^{\top} \cdots \times_{N} \mathbf{U}_{N}^{\top}-\mathcal{Y}_{j} \times{ }_{1} \mathbf{V}_{1}^{\top} \cdots \times \times_{N} \mathbf{V}_{N}^{\top}\right\|_{F}^{2} \tilde{w}_{i j}}
$$

where $\pi_{i}$ and $\pi_{j}$ denote the class labels of samples $i$ and $j, \bar{w}_{i j}$ denotes the similarity of intra-class gait data, and $\tilde{w}_{i j}$ denotes the similarity of inter-class gait data, which are defined as

$\bar{w}_{i j}=\left\{\begin{array}{l}1, \text { if } i \in N_{k_{1}}^{+}(j) \text { or } j \in N_{k_{1}}^{+}(i) \\ 0, \text { else }\end{array}, \tilde{w}_{i j}=\left\{\begin{array}{l}1, \text { if } i \in N_{k_{2}}^{-}(j) \text { or } j \in N_{k_{2}}^{-}(i) \\ 0, \text { else }\end{array}\right.\right.$,

where $N_{k_{1}}^{+}(\cdot)$ denotes a set of $k_{1}$ intra-class nearest neighbors, and $N_{k_{2}}^{-}(\cdot)$ denotes a set of $k_{2}$ inter-class nearest neighbors.

Like CMLP, the alternating projection optimization procedure can also decompose (16) into $N$ sub-optimization problems as follows

$\arg \min _{\mathbf{U}_{n}, \mathbf{V}_{n}} J\left(\mathbf{U}_{n}, \mathbf{V}_{n}\right)=\frac{\sum_{\pi_{i}=\pi_{j}}\left\|\mathbf{U}_{n}^{\top} \mathbf{X}_{i(n)} \tilde{\mathbf{U}}_{n}-\mathbf{V}_{n}^{\top} \mathbf{Y}_{j(n)} \tilde{\mathbf{V}}_{n}\right\|_{F}^{2} \bar{w}_{i j}}{\sum_{\pi_{i} \neq \pi_{j}}\left\|\mathbf{U}_{n}^{\top} \mathbf{X}_{i(n)} \tilde{\mathbf{U}}_{n}-\mathbf{V}_{n}^{\top} \mathbf{Y}_{j(n)} \tilde{\mathbf{V}}_{n}\right\|_{F}^{2} \tilde{w}_{i j}}, n=1, \cdots, N$ 
Eq. (18) can be rewritten as

$$
\begin{aligned}
& \arg \min _{\mathbf{U}_{n}, \mathbf{V}_{n}} J\left(\mathbf{U}_{n}, \mathbf{V}_{n}\right)= \\
& \frac{\operatorname{Tr}\left(\left[\begin{array}{c}
\mathbf{U}_{n} \\
\mathbf{V}_{n}
\end{array}\right]^{\top}\left[\begin{array}{cc}
\mathbf{X}_{(n)} \tilde{\mathbf{U}}_{n} & \mathbf{0} \\
\mathbf{0} & \mathbf{Y}_{(n)} \tilde{\mathbf{V}}_{(n)}
\end{array}\right]\left[\begin{array}{cc}
\overline{\mathbf{D}}_{1} \otimes \mathbf{I} & -\overline{\mathbf{W}} \otimes \mathbf{I} \\
-\overline{\mathbf{W}}^{\top} \otimes \mathbf{I} & \overline{\mathbf{D}}_{2} \otimes \mathbf{I}
\end{array}\right]\left[\begin{array}{cc}
\mathbf{X}_{(n)} \tilde{\mathbf{U}}_{n} & \mathbf{0} \\
\mathbf{0} & \mathbf{Y}_{(n)} \tilde{\mathbf{V}}_{(n)}
\end{array}\right]^{\top}\left[\begin{array}{c}
\mathbf{U}_{n} \\
\mathbf{V}_{n}
\end{array}\right]\right)}{\operatorname{Tr}\left(\left[\begin{array}{c}
\mathbf{U}_{n} \\
\mathbf{V}_{n}
\end{array}\right]^{\top}\left[\begin{array}{cc}
\mathbf{X}_{(n)} \tilde{\mathbf{U}}_{n} & \mathbf{0} \\
\mathbf{0} & \mathbf{Y}_{(n)} \tilde{\mathbf{V}}_{(n)}
\end{array}\right]\left[\begin{array}{cc}
\tilde{\mathbf{D}}_{1} \otimes \mathbf{I} & -\tilde{\mathbf{W}} \otimes \mathbf{I} \\
-\tilde{\mathbf{W}}^{\top} \otimes \mathbf{I} & \tilde{\mathbf{D}}_{2} \otimes \mathbf{I}
\end{array}\right]\left[\begin{array}{cc}
\mathbf{X}_{(n)} \tilde{\mathbf{U}}_{n} & \mathbf{0} \\
\mathbf{0} & \mathbf{Y}_{(n)} \tilde{\mathbf{V}}_{(n)}
\end{array}\right]^{\top}\left[\begin{array}{c}
\mathbf{U}_{n} \\
\mathbf{V}_{n}
\end{array}\right]\right)}
\end{aligned}
$$

where $\overline{\mathbf{W}}$ and $\tilde{\mathbf{W}}$ are intra-class similarity matrix and inter-class penalty similarity matrix respectively and both of their $i$-th row $j$-th column elements are $\bar{w}_{i j}$ and $\tilde{w}_{i j}$. Four diagonal matrices $\overline{\mathbf{D}}_{1}, \overline{\mathbf{D}}_{2}, \tilde{\mathbf{D}}_{1}$ and $\tilde{\mathbf{D}}_{2}$ are

$$
\begin{aligned}
\overline{\mathbf{D}}_{1} & =\left[\begin{array}{ccc}
\sum_{j} \bar{w}_{1 j} & \mathbf{0} & \mathbf{0} \\
\mathbf{0} & \ddots & \mathbf{0} \\
\mathbf{0} & \mathbf{0} & \sum_{j} \bar{w}_{M j}
\end{array}\right], \overline{\mathbf{D}}_{2}=\left[\begin{array}{ccc}
\sum_{i} \bar{w}_{i 1} & \mathbf{0} & \mathbf{0} \\
\mathbf{0} & \ddots & \mathbf{0} \\
\mathbf{0} & \mathbf{0} & \sum_{i} \bar{w}_{i M}
\end{array}\right], \\
\tilde{\mathbf{D}}_{1} & =\left[\begin{array}{ccc}
\sum_{j} \tilde{w}_{1 j} & \mathbf{0} & \mathbf{0} \\
\mathbf{0} & \ddots & \mathbf{0} \\
\mathbf{0} & \mathbf{0} & \sum_{j} \tilde{w}_{M j}
\end{array}\right], \tilde{\mathbf{D}}_{2}=\left[\begin{array}{ccc}
\sum_{i} \tilde{w}_{i 1} & \mathbf{0} & \mathbf{0} \\
\mathbf{0} & \ddots & \mathbf{0} \\
\mathbf{0} & \mathbf{0} & \sum_{i} \tilde{w}_{i M}
\end{array}\right] .
\end{aligned}
$$

To simplify (19), two alignment matrices are defined as follows

$$
\overline{\mathbf{G}}=\left[\begin{array}{cc}
\overline{\mathbf{D}}_{1} \otimes \mathbf{I} & -\overline{\mathbf{W}} \otimes \mathbf{I} \\
-\mathbf{W}^{\top} \otimes \mathbf{I} & \overline{\mathbf{D}}_{2} \otimes \mathbf{I}
\end{array}\right], \tilde{\mathbf{G}}=\left[\begin{array}{cc}
\tilde{\mathbf{D}}_{1} \otimes \mathbf{I} & -\tilde{\mathbf{W}} \otimes \mathbf{I} \\
-\tilde{\mathbf{W}}^{\top} \otimes \mathbf{I} & \tilde{\mathbf{D}}_{2} \otimes \mathbf{I}
\end{array}\right] .
$$

Then, (19) reduces to

$$
\arg \min _{\mathbf{P}_{n}} J\left(\mathbf{P}_{n}\right)=\frac{\operatorname{Tr}\left(\mathbf{P}_{n}^{\top} \mathbf{Z}_{(n)} \overline{\mathbf{G}} \mathbf{Z}_{(n)}^{\top} \mathbf{P}_{n}\right)}{\operatorname{Tr}\left(\mathbf{P}_{n}^{\top} \mathbf{Z}_{(n)} \tilde{\mathbf{G}} \mathbf{Z}_{(n)}^{\top} \mathbf{P}_{n}\right)}
$$

Like CMLP, a regularizer $\tau \mathbf{I}$, which can be viewed as a small disturbance, can be also imposed on the item $\tilde{\mathbf{G}}$ to avoid over fitting. Then we have the following criterion

$$
\arg \min _{\mathbf{P}_{n}} J\left(\mathbf{P}_{n}\right)=\frac{\operatorname{Tr}\left(\mathbf{P}_{n}^{\top} \mathbf{Z}_{(n)} \overline{\mathbf{G}} \mathbf{Z}_{(n)}^{\top} \mathbf{P}_{n}\right)}{\operatorname{Tr}\left(\mathbf{P}_{n}^{\top} \mathbf{Z}_{(n)}(\tilde{\mathbf{G}}+\tau \mathbf{I}) \mathbf{Z}_{(n)}^{\top} \mathbf{P}_{n}\right)}
$$


The above problem can be converted to solving the generalized eigen-decomposition problem. As a summary, the iterative procedure for the projection of cross-view gaits with CMMF criterion is presented in Algorithm 2.

\subsection{Coupled multi-linear discriminant analysis criterion (CMDA)}

General Tensor Discriminant Analysis (GTDA) is a linear Discriminant analysis extended in the tensor space, which introduce supervised information into multi-linear analysis. Motivated by GTDA, the mode $n$ intra-class scatter and inter-class scatter matrices in the projected tensor space are defined as follows:

$$
\begin{aligned}
& J_{w}\left(\mathbf{U}_{n}, \mathbf{V}_{n}\right)=\sum_{c=1}^{C}\left(\sum_{i=1}^{N_{c}}\left\|\mathbf{U}_{n}^{\top} \mathbf{X}_{i(n)}^{(c)} \tilde{\mathbf{U}}_{n}-\mathbf{U}_{n}^{\top} \overline{\mathbf{X}}_{(n)}^{(c)} \tilde{\mathbf{U}}_{n}\right\|_{F}^{2}+\sum_{j=1}^{N_{c}}\left\|\mathbf{V}_{n}^{\top} \mathbf{Y}_{j(n)}^{(c)} \tilde{\mathbf{V}}_{n}-\mathbf{V}_{n}^{\top} \overline{\mathbf{Y}}_{(n)}^{(c)} \tilde{\mathbf{V}}_{n}\right\|_{F}^{2}\right), \\
& J_{b}\left(\mathbf{U}_{n}, \mathbf{V}_{n}\right)=\sum_{c=1}^{C}\left(N_{c}\left\|\mathbf{U}_{n}^{\top} \overline{\mathbf{X}}_{(n)}^{(c)} \tilde{\mathbf{U}}_{n}-\mathbf{U}_{n}^{\top} \overline{\mathbf{X}}_{(n)} \tilde{\mathbf{U}}_{n}\right\|_{F}^{2}+N_{c}\left\|\mathbf{V}_{n}^{\top} \overline{\mathbf{Y}}_{(n)}^{(c)} \tilde{\mathbf{V}}_{n}-\mathbf{V}_{n}^{\top} \overline{\mathbf{Y}}_{(n)} \tilde{\mathbf{V}}_{n}\right\|_{F}^{2}\right),
\end{aligned}
$$

where $\overline{\mathbf{X}}_{(n)}^{(c)}, \overline{\mathbf{Y}}_{(n)}^{(c)}$ are the means of samples from the class c from two views $\theta$

and $\vartheta$, respectively, $\overline{\mathbf{X}}_{(n)}$ and $\overline{\mathbf{Y}}_{(n)}$, are the means of total data sets from two views $\theta$ and $\vartheta$ respectively.

$\overline{\mathbf{X}}_{(n)}^{(c)}=\frac{1}{N_{c}} \sum_{i=1}^{N_{c}} \mathbf{X}_{i(n)}^{(c)}, \overline{\mathbf{Y}}_{(n)}^{(c)}=\frac{1}{N_{c}} \sum_{j=1}^{N_{c}} \mathbf{Y}_{j(n)}^{(c)}, \overline{\mathbf{X}}_{(n)}=\frac{1}{C} \sum_{c=1}^{C} \overline{\mathbf{X}}_{(n)}^{(c)}, \overline{\mathbf{Y}}_{(n)}=\frac{1}{C} \sum_{c=1}^{C} \overline{\mathbf{Y}}_{(n)}^{(c)}$.

Again we wish to construct a large scalar when the mode $n$ intra-class scatter is small and the mode $n$ inter-class scatter is large.

$$
\left\{\mathbf{U}_{n}^{*}, \mathbf{V}_{n}^{*}\right\}=\underset{\mathbf{U}_{n}, \mathbf{V}_{n}}{\arg \max } \frac{J_{b}\left(\mathbf{U}_{n}, \mathbf{V}_{n}\right)}{J_{w}\left(\mathbf{U}_{n}, \mathbf{V}_{n}\right)}=\frac{\sum_{c=1}^{C}\left(N_{c}\left\|\mathbf{U}_{n}^{\top} \overline{\mathbf{X}}_{(n)}^{(c)} \tilde{\mathbf{U}}_{n}-\mathbf{U}_{n}^{\top} \overline{\mathbf{X}}_{(n)} \tilde{\mathbf{U}}_{n}\right\|_{F}^{2}+N_{c}\left\|\mathbf{V}_{n}^{\top} \overline{\mathbf{Y}}_{(n)}^{(c)} \tilde{V}_{n}-\mathbf{V}_{n}^{\top} \overline{\mathbf{Y}}_{(n)} \tilde{V}_{n}\right\|_{F}^{2}\right)}{\sum_{c=1}^{C}\left(\sum_{i=1}^{N_{c}}\left\|\mathbf{U}_{n}^{\top} \mathbf{X}_{i(n)}^{(c)} \tilde{\mathbf{U}}_{n}-\mathbf{U}_{n}^{\top} \overline{\mathbf{X}}_{(n)}^{(c)} \tilde{\mathbf{U}}_{n}\right\|_{F}^{2}+\sum_{j=1}^{N_{c}}\left\|\mathbf{V}_{n}^{\top} \mathbf{Y}_{j(n)}^{(c)} \tilde{\mathbf{V}}_{n}-\mathbf{V}_{n}^{\top} \overline{\mathbf{Y}}_{(n)}^{(c)} \tilde{\mathbf{V}}_{n}\right\|_{F}^{2}\right)}
$$

(26) can be rewritten as

$$
\begin{aligned}
& \arg \min _{\mathbf{U}_{n}, \mathbf{V}_{n}} J\left(\mathbf{U}_{n}, \mathbf{V}_{n}\right)=
\end{aligned}
$$

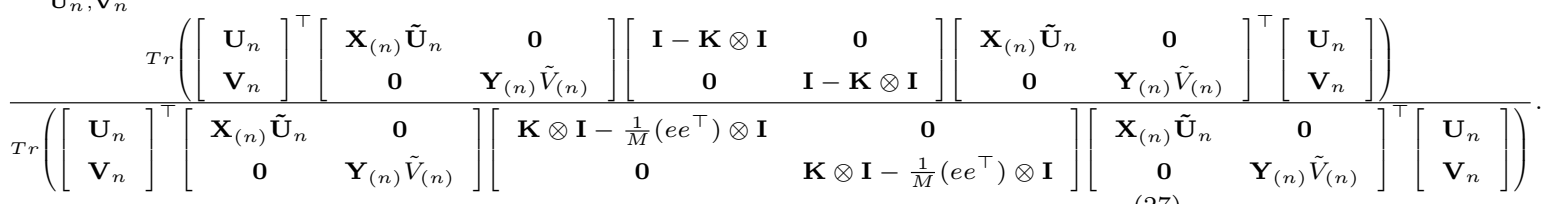

240 The detailed mathematical deductions of (27) are put into the Appendix D. 


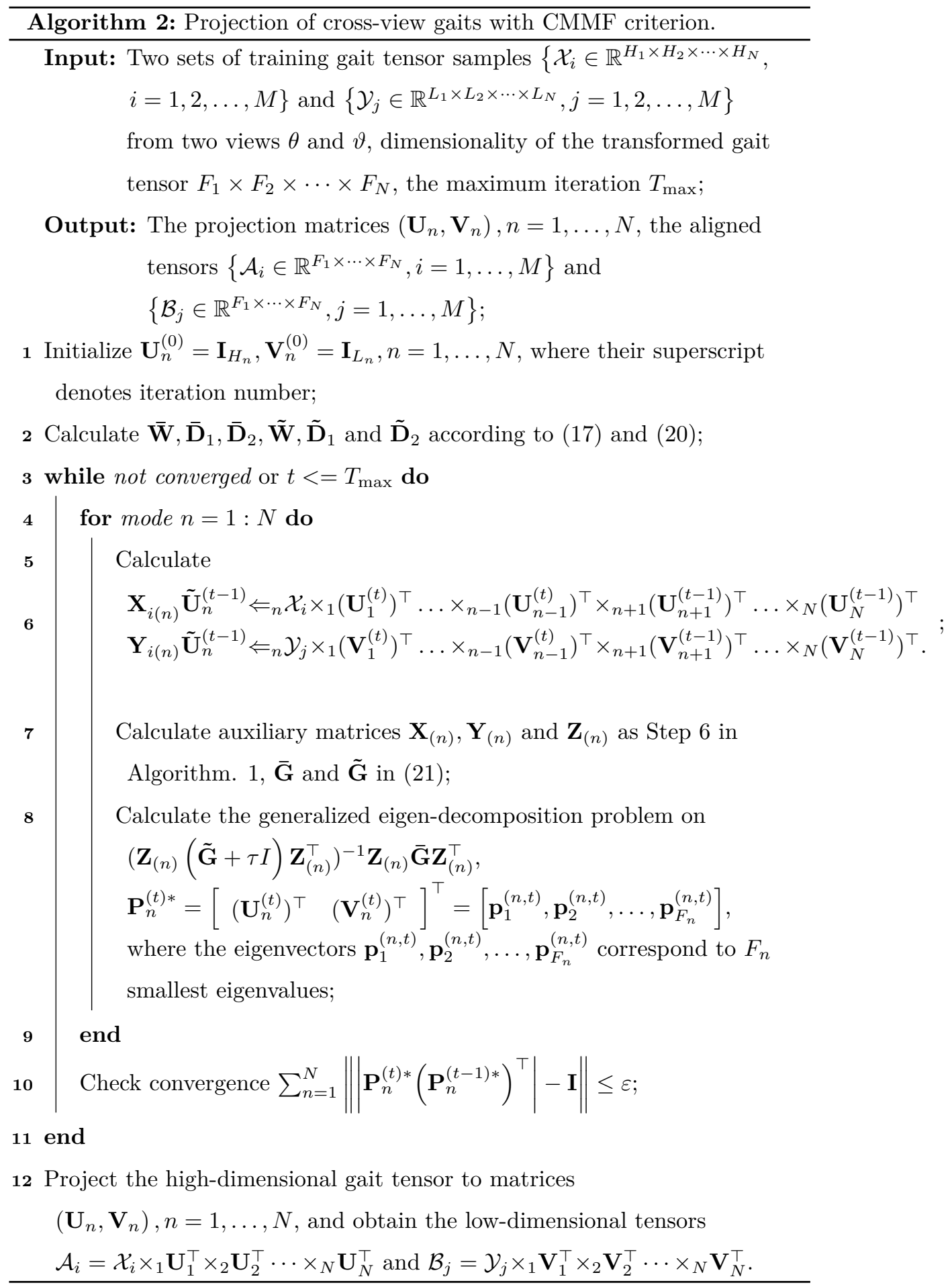


To simplify (27), several auxiliary matrices are defined as follows

$$
\begin{aligned}
& \mathbf{P}_{n}=\left[\begin{array}{c}
\mathbf{U}_{n} \\
\mathbf{V}_{n}
\end{array}\right], \mathbf{Z}_{(n)}=\left[\begin{array}{cc}
\mathbf{X}_{(n)} \tilde{\mathbf{U}}_{n} & \mathbf{0} \\
\mathbf{0} & \mathbf{Y}_{(n)} \tilde{V}_{n}
\end{array}\right], \mathbf{G}=\left[\begin{array}{cc}
\mathbf{I}-\mathbf{K} \otimes \mathbf{I} & \mathbf{0} \\
\mathbf{0} & \mathbf{I}-\mathbf{K} \otimes \mathbf{I}
\end{array}\right], \\
& \mathbf{G}^{\prime}=\left[\begin{array}{cc}
\mathbf{K} \otimes \mathbf{I}-\frac{1}{M}\left(\mathbf{e e}^{\top}\right) \otimes \mathbf{I} & \mathbf{0} \\
\mathbf{0} & \mathbf{K} \otimes \mathbf{I}-\frac{1}{M}\left(\mathbf{e e}^{\top}\right) \otimes \mathbf{I}
\end{array}\right] .
\end{aligned}
$$

Then, (27) reduces to

$$
\arg \min _{\mathbf{P}_{n}} J\left(\mathbf{P}_{n}\right)=\frac{\operatorname{Tr}\left(\mathbf{P}_{n}^{\top} \mathbf{Z}_{(n)} \mathbf{G} \mathbf{Z}_{(n)}^{\top} \mathbf{P}_{n}\right)}{\operatorname{Tr}\left(\mathbf{P}_{n}^{\top} \mathbf{Z}_{(n)} \mathbf{G}^{\prime} \mathbf{Z}_{(n)}^{\top} \mathbf{P}_{n}\right)} .
$$

The above problem can be converted to solve the generalized eigen-decomposition problem. As a summary, the iterative procedure for the projection of cross-view gaits with CMDA criterion is presented in Algorithm 3 .

\subsection{Analysis}

Complexity analysis. For all of the three criteria, the time complexity includes three aspects: $n$-mode projection in step 5 of Algorithm 11 and 2, step 4 in Algorithm 3, the scatter calculation and general eigenvalue decomposition.

In each iteration, the time complexity of the $n$-mode projection and general eigenvalue decomposition can be respectively computed by $O\left(M \sum_{n=1}^{N} H_{n}^{2} \widetilde{H}_{n}+\right.$ $\left.L_{n}^{2} \widetilde{L}_{n}\right)$ and $O\left(\sum_{n=1}^{N}\left(H_{n}+L_{n}\right)^{3}\right)$ for all the three variations, where $\widetilde{H}_{n}=$ $\Pi_{i=1, i \neq n}^{N} H_{i}$ and $\widetilde{L}_{n}=\Pi_{i=1, i \neq n}^{N} L_{i}$. For CMLP, the complexity to compute the scatter matrices in the optimization procedure is $O\left(\sum_{i=1}^{N} 2 M K_{n}\left(H_{n}+\right.\right.$ $\left.\left.{ }_{25} L_{n}\right)\left(2 M K_{n}+H_{n}+L_{n}\right)\right)$. CMMF and CMDA considers both inter-class scatter and intra-class scatter, which doubles the computation cost, i.e., $O\left(\sum_{i=1}^{N} 4 M K_{n}\left(H_{n}+\right.\right.$ $\left.\left.L_{n}\right)\left(2 M K_{n}+H_{n}+L_{n}\right)\right)$. For simplicity, we assume that $H_{n}=L_{n}=\left(\prod_{n=1}^{N} H_{n}\right)^{1 / n}=$ $\left(\prod_{n=1}^{N} L_{n}\right)^{1 / n}=I$, thus the computing complexity of CMLP can be denoted as $\left.\left.O\left(2 M N \cdot I^{(N+1)}+8 N \cdot I^{3}+8 M N I\left(M \cdot F^{(} N-1\right)+I\right) * F^{(} N-1\right)\right)$ where it 260 is assumed the dimensionality of transformed tensor $\forall F_{n}=F, n=1,2, \ldots, N$. Similarly, CMMF and CMDA double the computational complexity. 


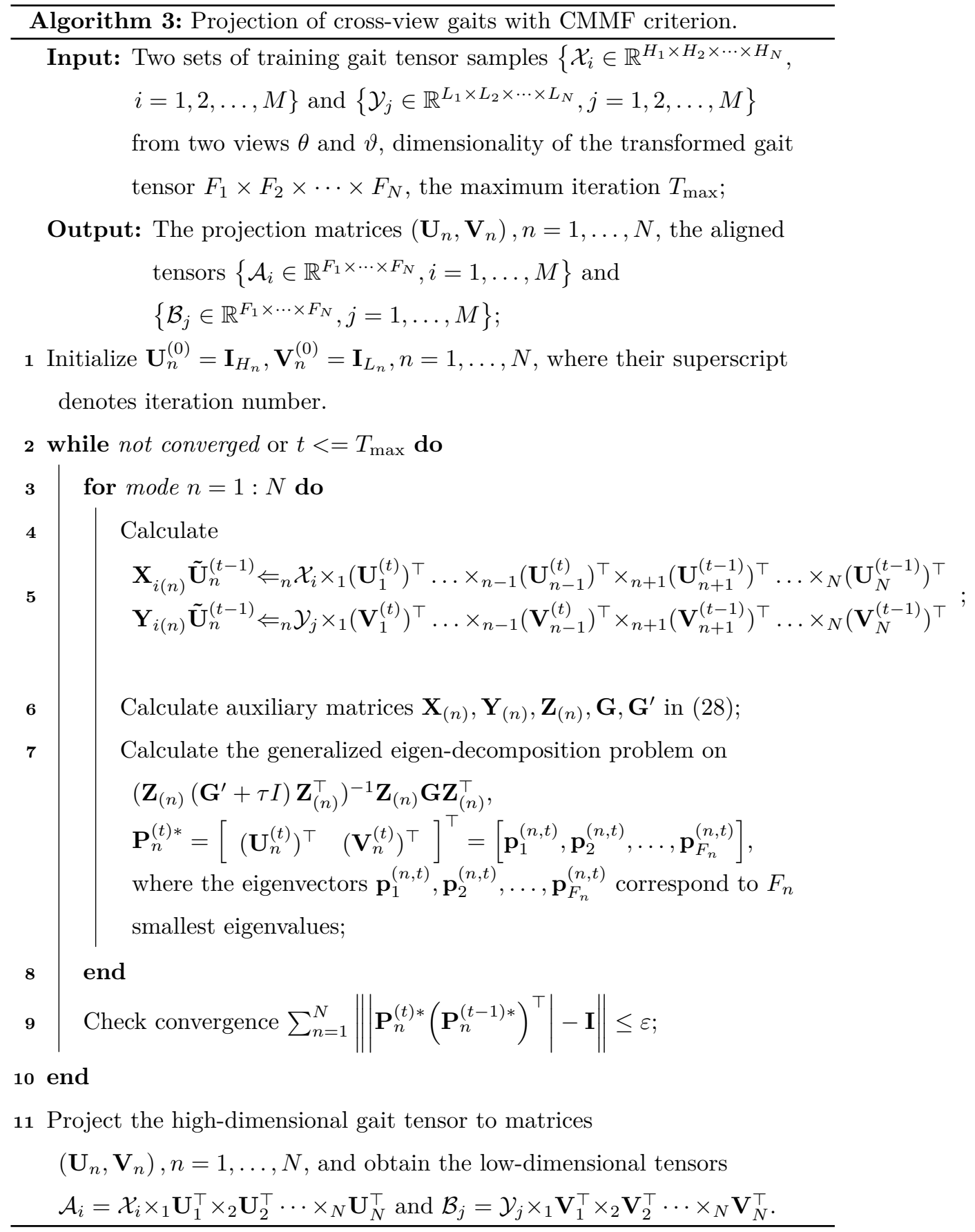


Relationship analysis. All of the proposed three variations under the unified framework aim to learn a shared multi-linear subspace in which the data biases caused by view differences are alleviated. Then, gait across different views

can be directly measured in the shared subspace. However, the three criteria construct different manifold structure in the subspace. As in Table 2, different alignment representation are embedded into the manifold graph, which makes the subspace discriminable. In detail, CMLP constructs a graph incorporating neighbourhood information across gait views spanned on the dataset. From perspective of Laplacian, we asymmetrically map the tensor data points from different gait views into a shared subspace by a couple of multi-linear projection matrices, which the multi-linear transformation optimally preserves local neighbourhood structure in the shared subspace. CMMF also blurs the data bias across views by optimally learning asymmetrical projections based on local neighbourhood information. However, CMMF is a multi-linear tensor discriminative model, which encodes both the intra-class compactness and inter-class separability with local neighbourhood relationships in the manifold. CMDA is also a discriminative model which make the samples from the same class compact and samples from the different class separate in the shared subspace. In contrast to CMLP and CMMF, CMDA encodes not only local neighbourhood information but also global relationships between classes. Though the three criteria follow the same framework, each of them build unique manifold.

\section{Experiments}

In this section, the effectiveness of the proposed framework is assessed by extensive experiments conducted on two databases: (1) CASIA(B) gait database and (2) OU-ISIR large population gait database. Videos/images in both databases are collected from multi-view cameras; therefore they are most applicable to evaluate the performance of cross-view gait recognition. For each gait sequence, we use dual-ellipse fitting approach [53] to detect gait periodicity. Then, we adopt gait energy image (GEI) [54] as the gait feature in a gait cycle. After that, 


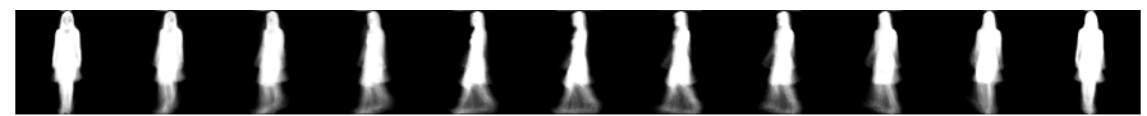

Figure 1: GEIs from the CASIA(B) database.

GaborD-based, GaborS-based and GaborSD-based gait representations [45] are used as recognized feature. GaborD and GaborS features are respectively obtained by the direction summation and scale summation of Gabor features, and GaborSD feature is obtained by both direction and scale summation of Gabor

features. For these three gait representations, the dimension of MPCA is chosen according to a $98 \%$ energy criterion as in [43]. Furthermore, the proposed three criteria of tensorial coupled mappings are used for extracting features. Finally, the nearest neighbor classifier is used for classification.

\subsection{Databases}

\subsubsection{CASIA(B) gait database}

The CASIA(B) gait database contains 13640 sequences of 124 subjects. For each subject, gaits are recorded by the cameras from 11 views, i.e. $0^{\circ}, 18^{\circ}, 36^{\circ}$, $54^{\circ}, 72^{\circ}, 90^{\circ}, 108^{\circ}, 126^{\circ}, 144^{\circ}, 162^{\circ}$, and $180^{\circ}$. There are 10 gait sequences for each subject: 6 samples under normal condition, 2 samples walking with coats and 2 samples carrying bags. Since the samples of walking with coats and carrying bags are too limited to calculate intra-class scatter, all the 6 samples containing normal walk for each subject are selected for the experiments in this paper. These normal walk samples are divided into training and testing sets, and the first 64 subjects are used for training and the rest 60 subjects are used to test the performance of gait recognition approaches under the view change. All the GEIs are cropped and normalized to $64 \times 64$ pixels. Figure 1 shows the GEIs from 11 viewing angles. After Gabor filter with the above-mentioned 5 scales and 8 directions, the sizes of GaborD-based, GaborS-based and GaborSD-based gait features are $64 \times 64 \times 8,64 \times 64 \times 5$ and $64 \times 64$, respectively.

\subsubsection{OU-ISIR large population gait database}

The OU-ISIR large population gait database has been released recently. It contains 1912 subjects whose gait sequences are captured from 4 different ob- 


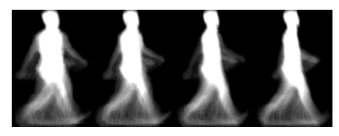

Figure 2: GEIs from the OU-ISIR database.

servation angles of $55^{\circ}, 65^{\circ}, 75^{\circ}$ and $85^{\circ}$. We randomly divide OU-ISIR gait database into two sets equally for 5 times. Therefore, 956 subjects compose the training set, and the rest 956 subjects make up the testing set. In our experiments, the size of GEIs is aligned to $64 \times 44$ pixels. Figure 2 shows GEIs from 4 different views. Compared with the CASIA(B) database, this database contains more subjects with wider range of age variations but narrower range of view variations. The sizes of GaborD-based, GaborS-based and GaborSD-

325 based gait features are $64 \times 44 \times 8,64 \times 44 \times 5$ and $64 \times 44$ respectively in our experiments.

\subsection{Performance evaluation}

\subsubsection{Evaluation on the CASIA(B) gait database}

In this section, we evaluate the effectiveness of the proposed GaborD-CMLP, GaborSD-CMLP, GaborSD-CMMF and GaborSD-CMDA using the CASIA(B) gait database. The numbers of iteration of CMLP, CMMF and CMDA are all set to 5. For fair comparison, the recognition accuracies with optimal parameters are reported. Table 3 illustrates the recognition rates of probe view $54^{\circ}$. It can be seen that when the difference increases between the probe and the gallery sample is $18^{\circ}$, the recognition rates of the proposed 9 methods, are above $90 \%$, and the recognition rate of each method is very close. In addition, the recognition rates decrease as the view difference between the gallery and probe views, but slow down the downward trend at a symmetrical gallery view 340 angle.

We compare the proposed methods with state-of-the-art cross-view gait recognition methods including GEI [54], CMCC[31], VTM+QM[23], SVD [24], SVR [32], MvDA [55] and GEI + Deep CNNs [40]. GEI is a kind of spatialtemporal template, which is the state-of-the-art feature representation in gait 
Table 3: Comparison on various methods on CASIA(B) under Probe view $54^{\circ}$ (the best record under each Gallery view is marked in bold, the second best is marked by underline '. '.)

\begin{tabular}{|c|c|c|c|c|c|c|c|c|c|c|}
\hline Gallery view & $0^{\circ}$ & $18^{\circ}$ & $36^{\circ}$ & $72^{\circ}$ & $90^{\circ}$ & $108^{\circ}$ & $126^{\circ}$ & $144^{\circ}$ & $162^{\circ}$ & $180^{\circ}$ \\
\hline GEI [54] & 0.04 & 0.09 & 0.30 & 0.22 & 0.18 & 0.17 & 0.38 & 0.19 & 0.02 & 0.03 \\
CMCC [31] & 0.24 & 0.65 & 0.97 & 0.95 & 0.63 & 0.53 & 0.48 & 0.34 & 0.23 & 0.22 \\
VTM+QM [23] & 0.21 & 0.67 & 0.96 & 0.97 & 0.70 & 0.66 & 0.39 & 0.33 & 0.20 & 0.22 \\
SVD [24] & 0.13 & 0.46 & 0.87 & 0.81 & 0.49 & 0.31 & 0.27 & 0.19 & 0.18 & 0.16 \\
SVR [32] & 0.22 & 0.64 & 0.95 & 0.93 & 0.59 & 0.51 & 0.42 & 0.27 & 0.20 & 0.21 \\
MvDA [55] & 0.28 & 0.70 & $\underline{0.98}$ & 0.97 & 0.72 & 0.68 & 0.53 & 0.42 & 0.25 & 0.28 \\
GEI + CNNs [40] & $\mathbf{0 . 5}$ & 0.67 & $\mathbf{0 . 9 9}$ & $\mathbf{0 . 9 9}$ & $\mathbf{0 . 9 3}$ & $\mathbf{0 . 9 0}$ & $\mathbf{0 . 7 9}$ & $\mathbf{0 . 7 3}$ & $\mathbf{0 . 6 3}$ & $\mathbf{0 . 6 2}$ \\
GaborD-CMLP & 0.37 & $\mathbf{0 . 7 8}$ & $\mathbf{0 . 9 9}$ & 0.97 & $\underline{0.82}$ & 0.69 & 0.64 & 0.55 & 0.33 & 0.32 \\
GaborD-CMMF & $\underline{0.43}$ & 0.72 & $\mathbf{0 . 9 9}$ & $\underline{0.98}$ & $\underline{0.82}$ & 0.71 & 0.66 & 0.56 & 0.37 & 0.30 \\
GaborD-CMDA & 0.31 & 0.51 & 0.92 & 0.86 & 0.57 & 0.58 & 0.53 & 0.30 & 0.25 & 0.25 \\
GaborS-CMLP & 0.38 & $\underline{0.74}$ & $\mathbf{0 . 9 9}$ & 0.97 & 0.79 & 0.73 & 0.73 & 0.51 & 0.38 & 0.33 \\
GaborS-CMMF & 0.42 & $\mathbf{0 . 7 8}$ & $\mathbf{0 . 9 9}$ & $\underline{0.98}$ & 0.81 & 0.76 & 0.65 & 0.53 & 0.37 & $\underline{0.35}$ \\
GaborS-CMDA & 0.31 & 0.66 & 0.96 & 0.95 & 0.74 & 0.61 & 0.61 & 0.42 & 0.34 & 0.29 \\
GaborSD-CMLP & 0.36 & 0.70 & $\underline{0.98}$ & 0.96 & $\underline{0.82}$ & 0.73 & 0.70 & $\underline{0.57}$ & 0.37 & 0.33 \\
GaborSD-CMMF & 0.37 & 0.72 & $\mathbf{0 . 9 9}$ & $\underline{0.98}$ & 0.81 & $\underline{0.77}$ & $\underline{0.76}$ & $\underline{0.57}$ & $\underline{0.42}$ & 0.33 \\
GaborSD-CMDA & 0.25 & 0.52 & 0.93 & 0.90 & 0.64 & 0.59 & 0.54 & 0.42 & 0.39 & 0.29 \\
\hline
\end{tabular}

345 recognition. We utilized GEI to characterize gait patterns in all of the compared approaches. For CMCC, the computing complexity includes four parts, i.e., bipartite graph modelling, bipartite graph multipartitioning, correlation optimization by CCA and linear approximation processes, which take $O\left(M I^{2}\right), O\left(I^{3}\right), O\left(\left(I^{\prime}\right)^{3}\right)$ and $O(M I)$, respectively, where $I^{\prime}$ is the dimension of the GEI segment. The complexity of VTM+QM and SVD is dominated by SVD factorization, which is $O\left(2 M^{2} I\right)$. For SVR, the complexity is related to the number of support vectors $S_{v}$, which is $O\left(S_{v}^{3}+M S_{v}^{2}+M I S_{v}\right)$ with upper bound $O\left(M^{2} I\right)$. For MvDA, computational costs are mainly from matrix inversion and eigenvalue decomposition, and both are $O\left(8 I^{3}\right)$. Table 3 tabulates comparison results under Probe view $54^{\circ}$. As shown in Table 3, the proposed methods consistently outperform other state-of-the-art methods. The average recognition rate of the proposed methods is $14 \%, 13 \%, 27 \%, 16 \%$ and $7 \%$ higher than CMCC, VTM+QM, SVD, SVR and MvDA. Particularly, the proposed methods achieve more remarkable increase under large view differences. Compared to GEI + CNNs, the proposed approaches achieve equivalent performances under small view variance, i.e., $18^{\circ}$. But, the performances of the proposed approaches are still posterior when the 
view differences are enlarged. Though GEI + CNNs achieves better performances, it demands large volume of labelled data and high computational costs which is not practical in gait recognition. In contrast, our proposed approaches overcome the drawbacks which aims at small size sample problem and reduces dependences on computational sources. Especially, our framework can achieve equal performance under small view variances.

\subsubsection{Evaluation on the OU-ISIR large population gait database}

Since OU-ISIR gait database contains two gait sequences per subject, each query subject's one angle view GEIs are used as gallery samples, and GEIs under other angle view are used as query samples. We repeat the experiment by swapping the samples in the training and testing sets, therefore, we test the recognition rates for 10 times for each cross-view as [55]. The average recognition rates over these 10 runs are reported in this paper.

We evaluate the accuracies of the proposed GaborD-CMLP, GaborD-CMMF, GaborD-CMDA, GaborS-CMLP, GaborS-CMMF, GaborS-CMDA, GaborSDCMLP, GaborSD-CMMF and GaborSD-CMDA, and also the effect of view angle variations. Table 4 illustrates the recognition rates with the gallery view and probe view of $55^{\circ}, 65^{\circ}, 75^{\circ}$ and $85^{\circ}$. We observe that the recognition rates decrease monotonically as the view difference between the gallery and probe views. The lowest recognition rate can reach more than $96 \%$ even when the maximum view difference is $30^{\circ}\left(85^{\circ}-55^{\circ}\right)$.

We also compare the proposed methods with those above-mentioned state-ofthe-art cross-view gait recognition methods using OU-ISIR database. Because the performance results of the proposed method are very close, the data is retained to the third digit after the decimal point, which is easier to distinguish between the pros and cons of them. Namely, we report the results accurate to one-thousandth for the OU-ISIR database. Table 4 presents the results in terms of recognition rate for the different methods. We can see that the proposed methods yield average increases of $13 \%, 17 \%, 19 \%, 20 \%$ and $5 \%$ as compared to CMCC, VTM+QM, SVD, SVR and MvDA. These results, again, corroborate 
Table 4: Comparison on various methods on OU-ISIR (the best record under each Gallery view is marked in bold, the second best is marked by underline '_.')

\begin{tabular}{|c|c|c|c|c|c|c|c|c|c|c|c|c|}
\hline Probe View $\left({ }^{\circ}\right)$ & \multicolumn{3}{|c|}{55} & \multicolumn{3}{|c|}{65} & \multicolumn{3}{|c|}{75} & \multicolumn{3}{|c|}{85} \\
\hline Gallery View $\left({ }^{\circ}\right)$ & 65 & 75 & 85 & 55 & 75 & 85 & 55 & 65 & 85 & 55 & 65 & 75 \\
\hline GEI [54] & 0.284 & 0.058 & 0.277 & 0.277 & 0.670 & 0.195 & 0.507 & 0.640 & 0.969 & 0.262 & 0.207 & 0.969 \\
\hline $\mathrm{CMCC} 31$ & 0.968 & 0.785 & 0.646 & $\underline{0.974}$ & 0.963 & 0.826 & 0.800 & 0.975 & 0.969 & 0.749 & 0.785 & 0.965 \\
\hline $\mathrm{VTM}+\mathrm{QM}[23$ & 0.941 & 0.704 & 0.491 & 0.957 & 0.966 & 0.785 & 0.756 & 0.971 & 0.964 & 0.555 & 0.838 & 0.978 \\
\hline SVD 24 & 0.932 & 0.704 & 0.523 & 0.923 & 0.936 & 0.771 & 0.774 & 0.940 & 0.947 & 0.523 & 0.763 & 0.925 \\
\hline SVR 32 & 0.936 & 0.710 & 0.531 & 0.940 & 0.943 & 0.720 & 0.753 & 0.943 & 0.941 & 0.511 & 0.711 & 0.938 \\
\hline MvDA 55 & 0.975 & 0.922 & 0.858 & $\underline{0.974}$ & $\underline{0.984}$ & 0.949 & 0.925 & 0.984 & 0.985 & 0.877 & 0.957 & $\underline{0.988}$ \\
\hline $\mathrm{GEI}+\mathrm{CNNs} 40$ & 0.983 & 0.960 & 0.805 & 0.963 & 0.973 & 0.833 & 0.942 & 0.978 & 0.851 & 0.900 & 0.960 & 0.984 \\
\hline GaborD-CMLP & 0.999 & 0.990 & 0.972 & 1.000 & 1.000 & $\underline{0.996}$ & 0.989 & 1.000 & 0.995 & 0.969 & $\underline{0.996}$ & 1.000 \\
\hline GaborD-CMMF & $\underline{0.998}$ & $\underline{0.991}$ & 0.970 & 1.000 & 1.000 & $\underline{0.996}$ & 0.990 & 1.000 & $\underline{0.999}$ & $\underline{0.971}$ & 0.994 & 1.000 \\
\hline GaborD-CMDA & $\underline{0.998}$ & 0.992 & 0.967 & 1.000 & 1.000 & 0.995 & $\underline{0.991}$ & 1.000 & 1.000 & 0.967 & 0.994 & 1.000 \\
\hline GaborS-CMLP & 0.999 & 0.992 & $\underline{0.971}$ & 1.000 & 1.000 & 0.997 & 0.993 & 1.000 & 1.000 & 0.972 & $\underline{0.996}$ & 1.000 \\
\hline GaborS-CMMF & 0.999 & 0.992 & 0.972 & 1.000 & 1.000 & 0.997 & 0.993 & 1.000 & 1.000 & 0.970 & 0.997 & 1.000 \\
\hline GaborS-CMDA & $\underline{0.998}$ & 0.989 & 0.968 & 1.000 & 1.000 & 0.997 & 0.990 & 1.000 & 1.000 & 0.963 & 0.997 & 1.000 \\
\hline GaborSD-CMLP & $\underline{0.998}$ & 0.989 & $\underline{0.971}$ & 1.000 & 1.000 & 0.997 & 0.975 & 1.000 & 1.000 & 0.970 & 0.993 & 1.000 \\
\hline GaborSD-CMMF & 0.999 & $\underline{0.991}$ & 0.965 & 1.000 & 1.000 & $\underline{0.996}$ & 0.987 & $\underline{0.999}$ & 1.000 & 0.966 & 0.994 & 1.000 \\
\hline GaborSD-CMDA & $\underline{0.998}$ & 0.984 & 0.967 & 1.000 & 1.000 & 0.994 & 0.988 & 1.000 & 1.000 & 0.964 & 0.994 & 1.000 \\
\hline
\end{tabular}

the useful tensor representation framework in addressing the cross-view gait recognition. It worth to point out that the proposed approaches achieve slightly prior performance compared to GEI + CNNs. This verifies the conclusion on

395 CASIA(B) dataset that the proposed framework is effective when view difference is relevant small.

\subsubsection{Ablation Study}

In this section, we evaluate the effectiveness of each component, i.e., Gaborbased features and tensor-based coupled metric learning framework. Figure 4003 compares identification accuracies versus various tensorial coupled mapping methods using GEI and Gabor-based features on OU-ISIR large population gait dataset. We show the recognition results of various gallery views under fixed probe view $75^{\circ}$ in sub-figure (a)-(c). From the figures, it can be seen that Gaborbased features achieve superior performances than GEI in most cases when the tensorial coupled mapping approach is fixed. This verifies that Gabor-based feature features boost the performances compared vanilla GEI. It is reasonable since Gabor-based features are learned from GEIs and sensitive to gait pattern 


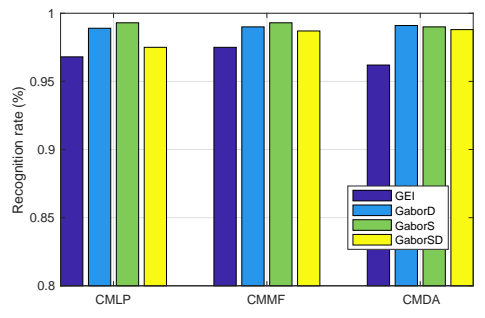

(a) Gallery view $55^{\circ}$

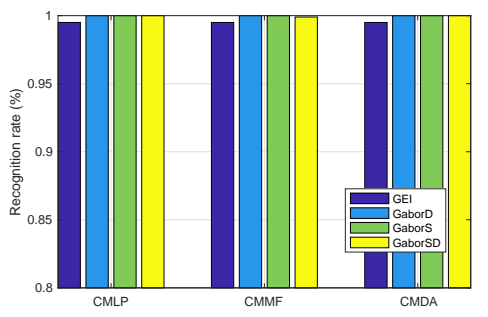

(b) Gallery view $65^{\circ}$

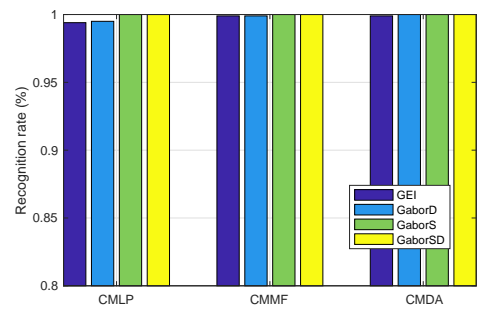

(c) Gallery view $85^{\circ}$

Figure 3: A comparison of different features on the OU-ISIR database.

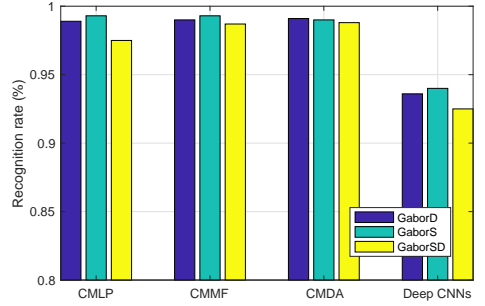

(a) Gallery view $55^{\circ}$

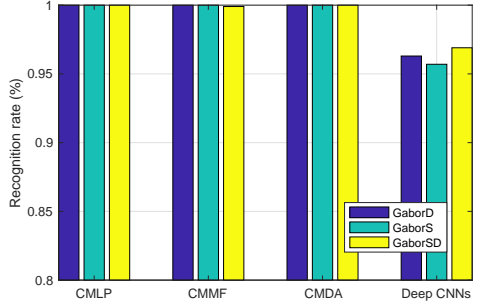

(b) Gallery view $65^{\circ}$

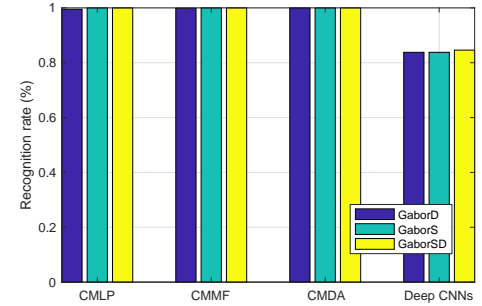

(c) Gallery view $85^{\circ}$

Figure 4: An illustration of the proposed tensorial coupled mapping framework with Gaborbased features on the OU-ISIR database.

variations. The conclusion is also verified in many deep learning studies [56, 57, and they prove that CNN learns Gabor features in shallow layers.

Figure 4 illustrates the effectiveness of the proposed tensorial coupled mapping framework. Sub-figure (a)-(c) show the recognition results of various gallery views, i.e., $55^{\circ}, 65^{\circ}$ and $85^{\circ}$, when the probe view is set to $75^{\circ}$. It is easy to observe that the proposed approaches under the unified tensorial coupled mapping framework outperform the deep CNNs with a large margin when combining with Gabor-based features. It demonstrates the effectiveness of our proposed tensorial coupled mapping framework. In another aspects, it exposes the shortage of deep CNNs that it is easy to be overfitting when the training data is limited. In particular, gait data is simple which also restricts the depth of deep CNNs [40]. It is worth to noting that GEI + CNNs achieves better performance than Gabor-based features + CNNs as in Table 4 . We believe that the reason is two-fold: one is that data-driven learned filters using deep CNNs are more 
appropriate than hand-crafted filters using Gabor; the other one is that it is easier to overfit for Gabor-based features than GEI when using the same depth CNN framework, because the deep CNNs learn Gabor features in shallow layers 425 56.

Thus it is appropriate to combine Gabor-based features and the proposed tensorial coupled mapping framework together. Each component improves performance of the whole gait recognition system.

\subsection{Discussion}

Based on the comparative experiments on the CASIA(B) and OU-ISIR gait databases, we discuss and analyze the effects of Gabor gait representation and three criteria of tensorial coupled mappings.

(1) The experiments in Section 5.2 show that the GaborS-based representation performs slightly better than GaborD-based representations which somewhat outperforms the GaborSD representation for cross-view gait recognition. This observation is consistent with [45]. GaborS and GaborD benefit cross-view gait recognition with Gabor functions over scales and directions representation.

(2) CMLP criterion has the ability to learn the essential gait manifold structure. By minimizing the distance between gait tensor data under two different observation angles for the identical subject, simultaneously suppressing the similarity of different subjects, as defined in (10), CMLP algorithm can enlarge the discrimination between different subjects, and meanwhile compact the variations of the same subject. Therefore, GaborD-CMLP, GaborS-CMLP and GaborSD-CMLP generally achieve good performance.

(3) CMMF criterion minimizes the ratio of intra-class similarity to the interclass similarity, which ensures the intra-class compactness and inter-class separability. Compared with CMLP criterion, CMMF criterion relies on Fisher discrimination which is more conducive to classification. Therefore, CMMF criterion yields better recognition performance than CMLP criterion. 
(4) Due to the lack of constrained relationship between cross-view gait tensor data, CMDA criterion is worst among the three criteria. Still, it is far superior to other state-of-the-art methods.

\section{Appendix A. Similarity between $\mathcal{A}_{i}$ and $\mathcal{B}_{i}$}

$$
\begin{aligned}
& \operatorname{sim}\left(\mathcal{A}_{i}, \mathcal{B}_{j}\right)=\left\|\mathcal{A}_{i}-\mathcal{B}_{j}\right\|_{F}^{2} w_{i j} \\
& =\operatorname{Tr}\left(\left(\mathbf{U}_{n}^{\top} \mathbf{X}_{i(n)} \tilde{\mathbf{U}}_{n}-\mathbf{V}_{n}^{\top} \mathbf{Y}_{j(n)} \tilde{\mathbf{V}}_{n}\right)\left(\mathbf{U}_{n}^{\top} \mathbf{X}_{i(n)} \tilde{\mathbf{U}}_{n}-\mathbf{V}_{n}^{\top} \mathbf{Y}_{j(n)} \tilde{\mathbf{V}}_{n}\right)^{\top} w_{i j}\right) \\
& =\operatorname{Tr}\left(\left[\begin{array}{c}
\mathbf{U}_{n} \\
\mathbf{V}_{n}
\end{array}\right]^{\top}\left[\begin{array}{cc}
\mathbf{X}_{i(n)} \tilde{\mathbf{U}}_{n} & \mathbf{0} \\
\mathbf{0} & \mathbf{Y}_{j(n)} \tilde{\mathbf{V}}_{n}
\end{array}\right]\left[\begin{array}{cc}
w_{i j} \cdot \mathbf{I} & -w_{i j} \cdot \mathbf{I} \\
-w_{i j} \cdot \mathbf{I} & w_{i j} \cdot \mathbf{I}
\end{array}\right]\left[\begin{array}{cc}
\mathbf{X}_{i(n)} \tilde{\mathbf{U}}_{n} & \mathbf{0} \\
\mathbf{0} & \mathbf{Y}_{j(n)} \tilde{\mathbf{V}}_{n}
\end{array}\right]\left[\begin{array}{c}
\mathbf{U}_{n} \\
\mathbf{V}_{n}
\end{array}\right]\right)
\end{aligned}
$$

\section{Appendix B. Derivation of mode- $n$ objective function of (11)}

The mode- $n$ objective function of (11) is obtained as follows: 


$$
\begin{aligned}
& \arg \min _{\mathbf{U}_{n}, \mathbf{V}_{n}} J\left(\mathbf{U}_{n}, \mathbf{V}_{n}\right)=\sum_{i, j}\left\|\mathbf{U}_{n}^{\top} \mathbf{X}_{i(n)} \tilde{\mathbf{U}}_{n}-\mathbf{V}_{n}^{\top} \mathbf{Y}_{j(n)} \tilde{\mathbf{V}}_{n}\right\|_{F}^{2} w_{i j} \\
& { }^{475}=\operatorname{Tr}\left(\sum_{i, j}\left[\begin{array}{c}
\mathbf{U}_{n} \\
\mathbf{V}_{n}
\end{array}\right]^{\top}\left[\begin{array}{c}
\mathbf{X}_{i(n)} \tilde{\mathbf{U}}_{n} \\
-\mathbf{Y}_{j(n)} \tilde{\mathbf{V}}_{n}
\end{array}\right]\left[\begin{array}{c}
\mathbf{X}_{i(n)} \tilde{\mathbf{U}}_{n} \\
-\mathbf{Y}_{j(n)} \tilde{\mathbf{V}}_{n}
\end{array}\right]^{\top}\left[\begin{array}{c}
\mathbf{U}_{n} \\
\mathbf{V}_{n}
\end{array}\right] w_{i j}\right) \\
& =\operatorname{Tr}\left(\left[\begin{array}{c}
\mathbf{U}_{n} \\
\mathbf{V}_{n}
\end{array}\right]^{\top}\left[\begin{array}{cc}
\mathbf{X}_{(n)} \tilde{\mathbf{U}}_{n}\left(\mathbf{D}_{1} \otimes \mathbf{I}\right) \tilde{\mathbf{U}}_{n}^{\top} \mathbf{X}_{(n)}^{\top} & -\mathbf{X}_{(n)} \tilde{\mathbf{U}}_{n}(\mathbf{W} \otimes \mathbf{I}) \tilde{\mathbf{V}}_{n}^{\top} \mathbf{Y}_{(n)}^{\top} \\
-\mathbf{Y}_{(n)} \tilde{\mathbf{V}}_{n}\left(\mathbf{W}^{\top} \otimes \mathbf{I}\right) \tilde{\mathbf{V}}_{n}^{\top} \mathbf{Y}_{(n)}^{\top} & \mathbf{Y}_{(n)} \tilde{\mathbf{V}}_{n}\left(\mathbf{D}_{2} \otimes \mathbf{I}\right) \tilde{\mathbf{V}}_{n}^{\top} \mathbf{Y}_{(n)}^{\top}
\end{array}\right]\left[\begin{array}{c}
\mathbf{U}_{n} \\
\mathbf{V}_{n}
\end{array}\right]\right) \\
& =\operatorname{Tr}\left(\left[\begin{array}{c}
\mathbf{U}_{n} \\
\mathbf{V}_{n}
\end{array}\right]^{\top}\left[\begin{array}{cc}
\mathbf{X}_{(n)} \tilde{\mathbf{U}}_{n} & \mathbf{0} \\
\mathbf{0} & \mathbf{Y}_{(n)} \tilde{\mathbf{V}}_{n}
\end{array}\right]\left[\begin{array}{cc}
\mathbf{D}_{1} \otimes \mathbf{I} & -\mathbf{W} \otimes \mathbf{I} \\
-\mathbf{W}^{\top} \otimes \mathbf{I} & \mathbf{D}_{2} \otimes \mathbf{I}
\end{array}\right]\left[\begin{array}{cc}
\mathbf{X}_{(n)} \tilde{\mathbf{U}}_{n} & \mathbf{0} \\
\mathbf{0} & \mathbf{Y}_{(n)} \tilde{\mathbf{V}}_{n}
\end{array}\right]^{\top}\left[\begin{array}{c}
\mathbf{U}_{n} \\
\mathbf{V}_{n}
\end{array}\right]\right)
\end{aligned}
$$

\section{Appendix C. The solution of (15)}

(15) can be solved by a generalized eigen-decomposition of

$$
\mathbf{Z}_{(n)} \mathbf{G} \mathbf{Z}_{(n)}^{\top} \mathbf{p}=\lambda\left(\mathbf{Z}_{(n)} \mathbf{Z}_{(n)}^{\top}\right) \mathbf{p}
$$

Taking the eigenvectors $\mathbf{p}_{1}^{(n)}, \mathbf{p}_{2}^{(n)}, \ldots, \mathbf{p}_{F_{n}}^{(n)}$ corresponding to $F_{n}\left(F_{n} \leq \max \left(H_{n}, L_{n}\right)\right)$ smallest eigenvalues $\lambda_{1}^{(n)} \leq \lambda_{2}^{(n)} \leq \cdots \leq \lambda_{F_{n}}^{(n)}$, the optimal solution of (22) can be represented as

$$
\mathbf{P}_{n}=\left[\begin{array}{c}
\mathbf{U}_{n} \\
\mathbf{V}_{n}
\end{array}\right]=\left[\mathbf{p}_{1}^{(n)}, \mathbf{p}_{2}^{(n)}, \ldots, \mathbf{p}_{F_{n}}^{(n)}\right]
$$

$\mathbf{Z}_{(n)} \mathbf{Z}_{(n)}^{\top}$ is usually non-invertible. In order to eliminate the singularity and to avoid over fitting, a regularizer $\tau \mathbf{I}$ is imposed on the item $\mathbf{Z}_{(n)} \mathbf{Z}_{(n)}^{\top}$, where

$\mathbf{I} \in \mathbb{R}^{\left(H_{n}+L_{n}\right) \times\left(H_{n}+L_{n}\right)}$ and is a small positive constant, such as $\tau=10^{-6}$ used in this paper. $\mathbf{P}_{n}$ can be divided into two matrices $\mathbf{U}_{n}$ and $\mathbf{V}_{n}$. $\mathbf{U}_{n}$ corresponds to the 1st to $H_{n}$-th rows of $\mathbf{P}_{n}$ and $\mathbf{V}_{n}$ corresponds to the $\left(H_{n}+1\right)$-th to $\left(H_{n}+L_{n}\right)$-th rows of $\mathbf{P}_{n}$. 


\section{Appendix D. Derivation of the mode- $n$ objective function of (27)}

The denominator of (27) can be rewritten as

$$
\begin{aligned}
J_{w}\left(\mathbf{U}_{n}, \mathbf{V}_{n}\right)=\operatorname{Tr}\left(\begin{array}{c}
\left(\sum_{c=1}^{C} \sum_{i=1}^{M}\left(\mathbf{U}_{n}^{\top} \mathbf{X}_{i(n)}^{(c)} \tilde{\mathbf{U}}_{n}-\mathbf{U}_{n}^{\top} \overline{\mathbf{X}}_{(n)}^{(c)} \tilde{\mathbf{U}}_{n}\right)\left(\mathbf{U}_{n}^{\top} \mathbf{X}_{i(n)}^{(c)} \tilde{\mathbf{U}}_{n}-\mathbf{U}_{n}^{\top} \overline{\mathbf{X}}_{(n)}^{(c)} \tilde{\mathbf{U}}_{n}\right)^{\top}\right. \\
\left.+\sum_{c=1}^{C} \sum_{j=1}^{M}\left(\mathbf{V}_{n}^{\top} \mathbf{Y}_{j(n)}^{(c)} \tilde{\mathbf{V}}_{n}-\mathbf{V}_{n}^{\top} \overline{\mathbf{Y}}_{(n)}^{(c)} \tilde{\mathbf{V}}_{n}\right)\left(\mathbf{V}_{n}^{\top} \mathbf{Y}_{j(n)}^{(c)} \tilde{\mathbf{V}}_{n}-\mathbf{V}_{n}^{\top} \overline{\mathbf{Y}}_{(n)}^{(c)} \tilde{\mathbf{V}}_{n}\right)^{\top}\right)
\end{array}\right) \\
=\operatorname{Tr}\left\{\left[\begin{array}{c}
\mathbf{U}_{n} \\
\mathbf{V}_{n}
\end{array}\right]^{\top}\left[\begin{array}{cc}
\mathbf{S}_{1} & \mathbf{0} \\
\mathbf{0} & \mathbf{S}_{2}
\end{array}\right]\left[\begin{array}{c}
\mathbf{U}_{n} \\
\mathbf{V}_{n}
\end{array}\right]\right\},
\end{aligned}
$$

where $\mathbf{X}_{c(n)}=\left[\mathbf{X}_{1(n)}^{(c)}, \cdots, \mathbf{X}_{M(n)}^{(c)}\right]$, for $c=1, \cdots, C, \mathbf{X}_{(n)}=\left[\mathbf{X}_{1(n)}, \cdots, \mathbf{X}_{C(n)}\right]$,

$$
\begin{aligned}
\mathbf{K}_{i j} & =\left\{\begin{array}{cll}
\frac{1}{N_{c}} & \text { if } \mathcal{X}_{\mathbf{i}}\left(\mathcal{Y}_{\mathrm{i}}\right) \text { and } \mathcal{X}_{\mathbf{j}}\left(\mathcal{Y}_{\mathrm{j}}\right) \text { both belong to the } c-\text { th class } \\
0 & \text { else }
\end{array}\right. \\
\mathbf{S}_{1} & =\sum_{c=1}^{C} \sum_{i=1}^{N_{c}}\left(\mathbf{X}_{i(n)}^{(c)} \tilde{\mathbf{U}}_{n}-\overline{\mathbf{X}}_{(n)}^{(c)} \tilde{\mathbf{U}}_{n}\right)\left(\mathbf{X}_{i(n)}^{(c)} \tilde{\mathbf{U}}_{n}-\overline{\mathbf{X}}_{(n)}^{(c)} \tilde{\mathbf{U}}_{n}\right)^{\top} \\
& =\sum_{c=1}^{C}\left(\left(\mathbf{X}_{c(n)} \tilde{\mathbf{U}}_{n}\right)\left(\mathbf{X}_{c(n)} \tilde{\mathbf{U}}_{n}\right)^{\top}-\frac{1}{N_{c}}\left(\mathbf{X}_{c(n)} \tilde{\mathbf{U}}_{n}\left(\left(e_{c} e_{c}^{\top}\right) \otimes \mathbf{I}\right)\left(\mathbf{X}_{c(n)} \tilde{\mathbf{U}}_{n}\right)^{\top}\right)\right. \\
& =\mathbf{X}_{(n)} \tilde{\mathbf{U}}_{n}(\mathbf{I}-\mathbf{K} \otimes \mathbf{I})\left(\mathbf{X}_{(n)} \tilde{\mathbf{U}}_{n}\right)^{\top},
\end{aligned}
$$

$$
\begin{aligned}
\mathbf{S}_{2} & =\sum_{c=1}^{C} \sum_{j=1}^{N_{c}}\left(\mathbf{Y}_{j(n)}^{(c)} \tilde{\mathbf{V}}_{n}-\overline{\mathbf{Y}}_{(n)}^{(c)} \tilde{\mathbf{V}}_{n}\right)\left(\mathbf{Y}_{j(n)}^{(c)} \tilde{\mathbf{V}}_{n}-\overline{\mathbf{Y}}_{(n)}^{(c)} \tilde{\mathbf{V}}_{n}\right)^{\top} \\
& =\left(\mathbf{Y}_{(n)} \tilde{\mathbf{V}}_{n}\right)(\mathbf{I}-\mathbf{K} \otimes \mathbf{I})\left(\mathbf{Y}_{(n)} \tilde{\mathbf{V}}_{n}\right)^{\top}
\end{aligned}
$$

Thus, the alignment expression is obtained as $J_{w}\left(\mathbf{U}_{n}, \mathbf{V}_{n}\right)=$

${ }_{495} \operatorname{Tr}\left(\left[\begin{array}{c}\mathbf{U}_{n} \\ \mathbf{V}_{n}\end{array}\right]^{\top}\left[\begin{array}{cc}\mathbf{X}_{(n)} \tilde{\mathbf{U}}_{n} & \mathbf{0} \\ \mathbf{0} & \mathbf{Y}_{(n)} \tilde{\mathbf{V}}_{(n)}\end{array}\right]\left[\begin{array}{cc}\mathbf{I}-\mathbf{K} \otimes \mathbf{I} & 0 \\ 0 & \mathbf{I}-\mathbf{K} \otimes \mathbf{I}\end{array}\right]\left[\begin{array}{cc}\mathbf{X}_{(n)} \tilde{\mathbf{U}}_{n} & \mathbf{0} \\ \mathbf{0} & \mathbf{Y}_{(n)} \tilde{\mathbf{V}}_{(n)}\end{array}\right]^{\top}\left[\begin{array}{c}\mathbf{U}_{n} \\ \mathbf{V}_{n}\end{array}\right]\right)$

The numerator of (27) can be rewritten as

$$
\begin{aligned}
J_{b}\left(\mathbf{U}_{n}, \mathbf{V}_{n}\right)= & \operatorname{Tr}\left(\begin{array}{l}
\left(\sum_{c=1}^{C} M\left(\mathbf{U}_{n}^{\top} \overline{\mathbf{X}}_{(n)}^{(c)} \tilde{\mathbf{U}}_{n}-\mathbf{U}_{n}^{\top} \overline{\mathbf{X}}_{(n)} \tilde{\mathbf{U}}_{n}\right)\left(\mathbf{U}_{n}^{\top} \overline{\mathbf{X}}_{(n)}^{(c)} \tilde{\mathbf{U}}_{n}-\mathbf{U}_{n}^{\top} \overline{\mathbf{X}}_{(n)} \tilde{\mathbf{U}}_{n}\right)^{\top}\right. \\
\left.+\sum_{c=1}^{C} M\left(\mathbf{V}_{n}^{\top} \overline{\mathbf{Y}}_{(n)}^{(c)} \tilde{\mathbf{V}}_{n}-\mathbf{V}_{n}^{\top} \overline{\mathbf{Y}}_{(n)} \tilde{\mathbf{V}}_{n}\right)\left(\mathbf{V}_{n}^{\top} \overline{\mathbf{Y}}_{(n)}^{(c)} \tilde{\mathbf{V}}_{n}-\mathbf{V}_{n}^{\top} \overline{\mathbf{Y}}_{(n)} \tilde{\mathbf{V}}_{n}\right)^{\top}\right)
\end{array}\right) \\
= & \operatorname{Tr}\left\{\left[\begin{array}{c}
\mathbf{U}_{n} \\
\mathbf{V}_{n}
\end{array}\right]^{\top}\left[\begin{array}{cc}
\mathbf{C}_{1} & \mathbf{0} \\
\mathbf{0} & \mathbf{C}_{2}
\end{array}\right]\left[\begin{array}{c}
\mathbf{U}_{n} \\
\mathbf{V}_{n}
\end{array}\right]\right\},
\end{aligned}
$$


where

$$
\begin{aligned}
\mathbf{C}_{1} & =\sum_{c=1}^{C} N_{c}\left(\overline{\mathbf{X}}_{(n)}^{(c)} \tilde{\mathbf{U}}_{n}-\overline{\mathbf{X}}_{(n)} \tilde{\mathbf{U}}_{n}\right)\left(\overline{\mathbf{X}}_{(n)}^{(c)} \tilde{\mathbf{U}}_{n}-\overline{\mathbf{X}}_{(n)} \tilde{\mathbf{U}}_{n}\right)^{\top} \\
& =\sum_{c=1}^{C} \sum_{i . j=1}^{N_{c}} \frac{1}{N_{c}}\left(\mathbf{X}_{i(n)}^{(c)} \tilde{\mathbf{U}}_{n}\right)\left(\mathbf{X}_{j(n)}^{(c)} \tilde{\mathbf{U}}_{n}\right)^{\top}-M \overline{\mathbf{X}}_{(n)} \tilde{\mathbf{U}}_{n}\left(\overline{\mathbf{X}}_{(n)} \tilde{\mathbf{U}}_{n}\right)^{\top} \\
& =\mathbf{X}_{(n)}\left(\mathbf{K} \otimes \mathbf{I}-\frac{1}{M}\left(\mathbf{e} \mathbf{e}^{\top}\right) \otimes \mathbf{I}\right) \mathbf{X}_{(n)}^{\top}, \\
\mathbf{C}_{2} & =\sum_{c=1}^{C} N_{c}\left(\overline{\mathbf{Y}}_{(n)}^{(c)} \tilde{\mathbf{V}}_{n}-\overline{\mathbf{Y}}_{(n)} \tilde{\mathbf{V}}_{n}\right)\left(\overline{\mathbf{Y}}_{(n)}^{(c)} \tilde{\mathbf{V}}_{n}-\overline{\mathbf{Y}}_{(n)} \tilde{\mathbf{V}}_{n}\right)^{\top} \\
& =\mathbf{Y}_{(n)}\left(\mathbf{K} \otimes \mathbf{I}-\frac{1}{M}\left(\mathbf{e e}^{\top}\right) \otimes \mathbf{I}\right) \mathbf{Y}_{(n)}^{\top}
\end{aligned}
$$

Thus, the alignment expression is obtained as follows

500

$$
\operatorname{Tr}\left([ \begin{array} { c } 
{ [ \mathbf { U } _ { n } } \\
{ \mathbf { V } _ { n } }
\end{array} ] ^ { \top } [ \begin{array} { c c } 
{ \mathbf { X } _ { ( n ) } \tilde { \mathbf { U } } _ { n } } & { \mathbf { 0 } } \\
{ \mathbf { 0 } } & { \mathbf { Y } _ { ( n ) } \tilde { \mathbf { V } } _ { ( n ) } }
\end{array} ] \left[\begin{array}{c}
\mathbf{K} \otimes \mathbf{I}-\frac{1}{M}\left(\mathbf{e e}^{\top}\right) \otimes \mathbf{I} \\
\mathbf{0}
\end{array}\right.\right.
$$

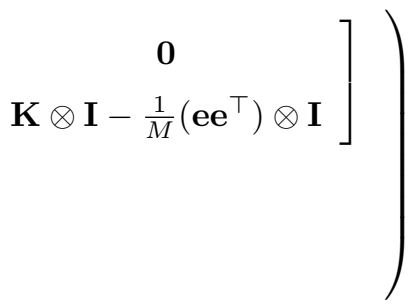

So the objective function for mode $n$ can be expressed as (27).

\section{Acknowledgment}

This project is supported by the National Key Research and Development

Program of China (Grant No. 2017YFC0803401), the Natural Science Foundation of China (Grant No. 61571275, 61573248, 61802267, 61201370), the Young Scholars Program of Shandong University, the Natural Science Foundation of Guangdong Province through the Grant 2017A030313367, and Shenzhen Municipal Science and Technology Innovation Council (Grant No. JCYJ20150324141711637, JCYJ20170302153434048).

\section{References}

[1] X. Ben, W. Meng, R. Yan, K. Wang, Kernel coupled distance metric learning for gait recognition and face recognition., Neurocomputing 120 (10) (2013) 577-589. 
[2] Y. He, J. Zhang, H. Shan, L. Wang, Multi-task gans for view-specific feature learning in gait recognition, IEEE Transactions on Information Forensics and Security 14 (1) (2019) 102-113.

[3] G. Ariyanto, M. S. Nixon, Model-based 3d gait biometrics, in: International Joint Conference on Biometrics, 2011, pp. 1-7.

[4] I. R. Vega, S. Sarkar, Statistical motion model based on the change of feature relationships: human gait-based recognition, IEEE Transactions on Pattern Analysis \& Machine Intelligence 25 (10) (2003) 1323-1328.

[5] C. Wang, J. Zhang, L. Wang, J. Pu, X. Yuan, Human identification using temporal information preserving gait template, IEEE Transactions on Pattern Analysis \& Machine Intelligence 34 (11) (2012) 2164-2176.

[6] L. Wang, T. Tan, H. Ning, W. Hu, Silhouette analysis-based gait recognition for human identification, IEEE Transactions on Pattern Analysis \& Machine Intelligence 25 (12) (2003) 1505-1518.

[7] Y. Guan, C. T. Li, F. Roli, On reducing the effect of covariate factors in gait recognition: A classifier ensemble method, IEEE Transactions on Pattern Analysis \& Machine Intelligence 37 (7) (2015) 1521-1528.

[8] S. Yu, D. Tan, T. Tan, Modelling the effect of view angle variation on appearance-based gait recognition, in: Asian Conference on Computer Vision, 2006, pp. 807-816.

535

[9] S. D. Choudhury, T. Tjahjadi, Robust view-invariant multiscale gait recognition, Pattern Recognition 48 (3) (2015) 798-811.

[10] J. Luo, J. Tang, T. Tjahjadi, X. Xiao, Robust arbitrary view gait recognition based on parametric $3 \mathrm{~d}$ human body reconstruction and virtual posture synthesis, Pattern Recognition 60 (2016) 361-377.

${ }_{540}$ [11] X. Ben, P. Zhang, W. Meng, R. Yan, M. Yang, W. Liu, H. Zhang, On the distance metric learning between cross-domain gaits, Neurocomputing 208 (2016) 153-164. 
[12] J. Lu, G. Wang, P. Moulin, Human identity and gender recognition from gait sequences with arbitrary walking directions, IEEE Transactions on Information Forensics \& Security 9 (1) (2014) 51-61.

[13] W. Zhang, X. Yu, X. He, Learning bidirectional temporal cues for videobased person re-identification, IEEE Transactions on Circuits and Systems for Video Technologydoi:10.1109/TCSVT . 2017.2718188.

[14] B. Du, W. Xiong, J. Wu, L. Zhang, L. Zhang, D. Tao, Stacked convolutional denoising auto-encoders for feature representation, IEEE Transactions on Cybernetics 47 (4) (2017) 1017-1027. doi:10.1109/TCYB.2016.2536638.

[15] C. Ding, D. Tao, Trunk-branch ensemble convolutional neural networks for video-based face recognition, IEEE transactions on pattern analysis and machine intelligence.

[16] S. Huang, Z. Xu, D. Tao, Y. Zhang, Part-stacked cnn for fine-grained visual categorization, in: Proceedings of the IEEE Conference on Computer Vision and Pattern Recognition, 2016, pp. 1173-1182.

[17] H. Lu, K. N. Plataniotis, A. N. Venetsanopoulos, A survey of multilinear subspace learning for tensor data, Pattern Recognition 44 (7) (2011) 15401551.

[18] S. Yu, D. Tan, T. Tan, A framework for evaluating the effect of view angle, clothing and carrying condition on gait recognition, in: International Conference on Pattern Recognition, 2006, pp. 441-444.

[19] H. Iwama, M. Okumura, Y. Makihara, Y. Yagi, The ou-isir gait database comprising the large population dataset and performance evaluation of gait recognition, IEEE Transactions on Information Forensics \& Security 7 (5) (2012) 1511-1521.

[20] G. Zhao, G. Liu, H. Li, M. Pietikainen, 3d gait recognition using multiple cameras, in: International Conference on Automatic Face and Gesture Recognition, 2006, pp. 529-534. 
[21] K. Sugiura, Y. Makihara, Y. Yagi, Gait identification based on multi-view observations using omnidirectional camera, in: Asian Conference on Computer Vision, 2007, pp. 452-461.

[22] J. Tang, J. Luo, T. Tjahjadi, F. Guo, Robust arbitrary-view gait recognition based on 3d partial similarity matching, IEEE Transactions on Image Processing 26 (1) (2016) 7-22.

[23] D. Muramatsu, Y. Makihara, Y. Yagi, View transformation model incorporating quality measures for cross-view gait recognition, IEEE Transactions on Cybernetics 46 (7) (2016) 1602-1615.

[24] W. Kusakunniran, Q. Wu, H. Li, J. Zhang, Multiple views gait recognition using view transformation model based on optimized gait energy image, in: IEEE International Conference on Computer Vision Workshops, 2010, pp. 1058-1064.

[25] W. Kusakunniran, Q. Wu, J. Zhang, H. Li, Multi-view gait recognition based on motion regression using multilayer perceptron, in: International Conference on Pattern Recognition, 2010, pp. 2186-2189.

[26] T. Connie, M. K. O. Goh, A. B. J. Teoh, A grassmannian approach to address view change problem in gait recognition, IEEE Transactions on Cybernetics 47 (6) (2017) 1395-1408.

[27] D. Muramatsu, A. Shiraishi, Y. Makihara, M. Z. Uddin, Y. Yagi, Gaitbased person recognition using arbitrary view transformation model, IEEE Transactions on Image Processing 24 (1) (2015) 140-154.

[28] S. Zheng, J. Zhang, K. Huang, R. He, T. Tan, Robust view transformation model for gait recognition, in: IEEE International Conference on Image Processing, 2011, pp. 2073-2076.

[29] Y. Makihara, R. Sagawa, Y. Mukaigawa, T. Echigo, Y. Yagi, Which reference view is effective for gait identification using a view transformation 
model?, in: Computer Vision and Pattern Recognition Workshop, 2006. CVPRW '06. Conference on, 2006, pp. 45-45.

[36] N. Liu, J. Lu, Y. P. Tan, Joint subspace learning for view-invariant gait recognition, IEEE Signal Processing Letters 18 (7) (2011) 431-434.

[37] X. Xing, K. Wang, T. Yan, Z. Lv, Complete canonical correlation analysis with application to multi-view gait recognition, Pattern Recognition 50 (2016) 107-117. 
38] J. Lu, Y. P. Tan, Uncorrelated discriminant simplex analysis for viewinvariant gait signal computing, Pattern Recognition Letters 31 (5) (2010) $382-393$.

[39] J. Lu, G. Wang, P. Moulin, Human identity and gender recognition from gait sequences with arbitrary walking directions, IEEE Transactions on Information Forensics \& Security 9 (1) (2014) 51-61.

[40] Z. Wu, Y. Huang, L. Wang, X. Wang, T. Tan, A comprehensive study on cross-view gait based human identification with deep cnns, IEEE Transactions on Pattern Analysis \& Machine Intelligence 39 (2) (2017) 209-226.

[41] N. Takemura, Y. Makihara, D. Muramatsu, T. Echigo, Y. Yagi, On in-

[43] H. Lu, K. N. Plataniotis, A. N. Venetsanopoulos, Mpca: Multilinear principal component analysis of tensor objects, IEEE Transactions on Neural Networks 19 (1) (2008) 18-39.

[44] H. Lu, K. N. Plataniotis, A. N. Venetsanopoulos, Uncorrelated multilinear principal component analysis for unsupervised multilinear subspace learning., IEEE Transactions on Neural Networks 20 (11) (2009) 1820-1836.

[45] D. Tao, X. Li, X. Wu, S. J. Maybank, General tensor discriminant analysis and gabor features for gait recognition, IEEE Transactions on Pattern Analysis and Machine Intelligence 29 (10) (2007) 1700-1715.

650

[42] K. Shiraga, Y. Makihara, D. Muramatsu, T. Echigo, Y. Yagi, Geinet: Viewinvariant gait recognition using a convolutional neural network, in: International Conference on Biometrics, 2016, pp. 1-8.

S. Yan, D. Xu, Q. Yang, L. Zhang, X. Tang, H. J. Zhang, Discriminant analysis with tensor representation, in: IEEE Computer Society Conference on Computer Vision and Pattern Recognition, 2005, pp. 526-532. 
[47] H. Lu, K. N. Plataniotis, A. N. Venetsanopoulos, Uncorrelated multilinear discriminant analysis with regularization and aggregation for tensor object recognition., IEEE Transactions on Neural Networks 20 (1) (2009) 103-23.

[48] X. Li, S. Lin, S. Yan, D. Xu, Discriminant locally linear embedding with high-order tensor data, IEEE Transactions on Systems Man \& Cybernetics Part B Cybernetics 38 (2) (2008) 342-352.

[49] X. Ben, P. Zhang, R. Yan, M. Yang, G. Ge, Gait recognition and microexpression recognition based on maximum margin projection with tensor representation, Neural Computing \& Applications 27 (8) (2016) 2629-2646.

[50] Y. L. Chen, C. T. Hsu, Multilinear graph embedding: Representation and regularization for images, IEEE Transactions on Image Processing 23 (2) (2014) 741-754.

665 [51] N. Zhao, L. Zhang, B. Du, L. Zhang, D. Tao, J. You, Sparse tensor discriminative locality alignment for gait recognition, in: International Joint Conference on Neural Networks, 2016, pp. 4489-4495.

[52] X. Ben, W. Meng, R. Yan, K. Wang, An improved biometrics technique based on metric learning approach, Neurocomputing 97 (2012) 44-51.

${ }_{670}$ [53] X. Ben, W. Meng, R. Yan, Dual-ellipse fitting approach for robust gait periodicity detection, Neurocomputing 79 (2012) 173-178.

[54] J. Han, B. Bhanu, Individual recognition using gait energy image, IEEE Transactions on Pattern Analysis \& Machine Intelligence 28 (2) (2005) $316-322$.

675 [55] A. Mansur, Y. Makihara, D. Muramatsu, Y. Yagi, Cross-view gait recognition using view-dependent discriminative analysis, in: IEEE International Joint Conference on Biometrics, 2014, pp. 1-8. doi:10.1109/BTAS. 2014. 6996272. 
[56] M. D. Zeiler, R. Fergus, Visualizing and understanding convolutional networks, in: European conference on computer vision, 2014, pp. 818-833.

[57] K. He, X. Zhang, S. Ren, J. Sun, Deep residual learning for image recognition, in: Proceedings of the IEEE conference on computer vision and pattern recognition, 2016, pp. 770-778. 